\title{
Random exponential attractor for second order non-autonomous stochastic lattice dynamical systems with multiplicative white noise in weighted spaces
}

Haijuan Su', Shengfan $\mathrm{Zhou}^{1 *}$ and Luyao $\mathrm{Wu}^{1}$

"Correspondence:

zhoushengfan@yahoo.com

${ }^{1}$ Department of Mathematics,

Zhejiang Normal University, Jinhua, P.R. China

\section{空 Springer}

\begin{abstract}
This paper is concerned with the random exponential attractor for second order non-autonomous stochastic lattice system with multiplicative white noise and unbounded nonlinearity. Firstly, we transfer the stochastic lattice system into a random lattice system without noise term whose solutions generate a continuous cocycle on a weighted space of infinite sequences. Then we present some sufficient conditions for the existence of a random exponential attractor for a continuous cocycle in the product weighted space of sequences, which improved the existing conditions. Finally, we prove the existence of a random exponential attractor for the considered system in weighted space of sequences.
\end{abstract}

MSC: $37 \mathrm{~L} 55 ; 60 \mathrm{H} 15 ; 35 \mathrm{~B} 40$

Keywords: Random exponential attractor; Stochastic lattice system; Multiplicative white noise; Cocycle; Weighted space of sequences

\section{Introduction}

It is well known that the dynamics of a random dynamical system can be determined by the random attractor. However, the speed of a random attractor attracting orbits is sometimes relatively slow and the dimension of a random attractor is maybe infinite [1]. To this end, Shirikyan and Zelik [2] introduced the random exponential attractor (a positively invariant measurable set with a finite fractal dimension and attracting any trajectory exponentially) for an autonomous random dynamical system. The random exponential attractor (if it exists) contains the random attractor with finite fractal dimension, which implies that the dynamics of a random dynamical system can be described by finite independent parameters. Recently, Zhou in [3] established the existence of a random exponential attractor for a continuous cocycle (non-autonomous random dynamical system) on a separable Banach space and the first order stochastic lattice system driven by linear multiplicative white noise.

(c) The Author(s) 2019. This article is distributed under the terms of the Creative Commons Attribution 4.0 International License (http://creativecommons.org/licenses/by/4.0/), which permits unrestricted use, distribution, and reproduction in any medium, provided you give appropriate credit to the original author(s) and the source, provide a link to the Creative Commons license, and indicate if changes were made. 
In this paper, we consider the following second order non-autonomous stochastic lattice system with multiplicative white noise and initial conditions: for every $\tau \in \mathbb{R}$ and $t>\tau$,

$$
\left\{\begin{array}{l}
\ddot{u}_{i}+\gamma(A \dot{u})_{i}+\alpha \dot{u}_{i}+(A u)_{i}+\lambda_{i} u_{i}+f_{i}\left(u_{i}, t\right)=g_{i}(t)+c u_{i} \circ \dot{W}(t), \quad i \in \mathbb{Z}, \\
u_{i}(\tau)=u_{0, i \tau}, \quad \dot{u}_{i}(\tau)=u_{1, i \tau}, \quad i \in \mathbb{Z},
\end{array}\right.
$$

where $i \in \mathbb{Z}, u=\left(u_{i}\right)_{i \in \mathbb{Z}}, \dot{u}=\left(\dot{u}_{i}\right)_{i \in \mathbb{Z}}, \alpha, c>0, \gamma \geq 0, \lambda_{i}>0, u_{i}, g_{i}(t), f_{i}\left(u_{i}, t\right) \in \mathbb{R}$, and $A$ is a non-negative linear coupled operator; $W(t)$ is a two-sided real-valued Wiener process on a probability space $(\Omega, \mathcal{F}, \mathbb{P})$, where $\Omega=\{\omega \in C(\mathbb{R}, \mathbb{R}): \omega(0)=0\}$, the Borel $\sigma$-algebra $\mathcal{F}$ on $\Omega$ is generated by the compact open topology, and $\mathbb{P}$ is the Wiener measure on $(\Omega, \mathcal{F})$ [4]; "o" in (1) denotes the Stratonovich sense in the stochastic term.

For the second order autonomous stochastic lattice system $(1)\left(f_{i}\left(u_{i}, t\right)=f_{i}\left(u_{i}\right), g_{i}(t)=g_{i}\right.$ independent of $t$ ) with multiplicative and additive white noise, the existence and upper semi-continuity of the random attractor have been studied by some authors; see [5-12]. But as is well known, there is no result concerning the dimension of random attractor and existence of random exponential attractors for second order autonomous and nonautonomous stochastic lattice system (1). Based on the ideas of $[3,5,9]$, in this paper, we aim to prove that under certain conditions, the system (1) possesses a random exponential attractor in weighted spaces of sequences, which implies that the corresponding autonomous and non-autonomous stochastic systems (1) have the random attractors with finite fractal dimension.

\section{Random exponential attractor for second order non-autonomous stochastic lattice system}

Let $\rho: \mathbb{Z} \rightarrow(0,+\infty), \rho_{i}=\rho(i)$ for $i \in \mathbb{Z}$ be a positive weight function. Write

$$
l_{\rho}^{2}=\left\{u=\left(u_{i}\right)_{i \in \mathbb{Z}}: u_{i} \in \mathbb{R}, \sum_{i \in \mathbb{Z}} \rho_{i}\left|u_{i}\right|^{2}<\infty\right\}
$$

with inner product $(u, v)_{\rho}=\sum_{i \in \mathbb{Z}} \rho_{i} u_{i} v_{i}$ and norm $\|v\|_{\rho}^{2}=(v, v)_{\rho}$ for $u=\left(u_{i}\right)_{i \in \mathbb{Z}}, v=\left(v_{i}\right)_{i \in \mathbb{Z}} \in$ $l_{\rho}^{2}$.

Consider the system (1), which can be written in the following vector form:

$$
\left\{\begin{array}{l}
\ddot{u}+\gamma A \dot{u}+\alpha \dot{u}+A u+\lambda u+f(u, t)=g(t)+c u \circ \dot{W}(t), \quad t>\tau, \\
u(\tau)=u_{\tau}, \quad \dot{u}(\tau)=u_{1 \tau}, \quad \tau \in \mathbb{R}
\end{array}\right.
$$

where $A \dot{u}=\left((A \dot{u})_{i}\right)_{i \in \mathbb{Z}}, A u=\left(A u_{i}\right)_{i \in \mathbb{Z}}, \lambda u=\left(\lambda_{i} u_{i}\right)_{i \in \mathbb{Z}}, f(u, t)=\left(f_{i}\left(u_{i}, t\right)\right)_{i \in \mathbb{Z}}, g(t)=\left(g_{i}(t)\right)_{i \in \mathbb{Z}}$, $u \circ \dot{W}(t)=\left(u_{i} \circ \dot{W}(t)\right)_{i \in \mathbb{Z}}$. The operator $A$ has a decomposition $A=\bar{D} D=D \bar{D}$, where $D$ is a bounded linear operator defined by

$$
(D u)_{i}=\sum_{l=-m_{0}}^{m_{0}} d_{l} u_{i+l}, \quad\left|d_{l}\right| \leq c_{0} \text { (constant), } \forall u=\left(u_{i}\right)_{i \in \mathbb{Z}}, m_{0} \in \mathbb{N},
$$

$\bar{D}$ is the adjoint of $D$ in $l^{2}=l_{\rho(i) \equiv 1, \forall i \in \mathbb{Z}}^{2}$

Let $i \in \mathbb{Z}, G_{i}(s, t)=\int_{0}^{s} f_{i}(r, t) d r, \bar{G}(u, t)=\sum_{i \in \mathbb{Z}} \rho_{i} G_{i}\left(u_{i}, t\right)$ and we make the following assumptions on $\rho_{i}, f_{i}, g_{i}, \lambda_{i}$ : 
(A1) $0<\bar{\rho} \leq \rho_{i}=\rho(i) \leq a_{0}<+\infty, c_{1} \rho(i) \leq \rho(i \pm 1) \leq c_{2} \rho(i),|\rho(i \pm 1)-\rho(i)| \leq c_{3} \rho(i)$, $\forall i \in \mathbb{Z}$, for some positive constants $\bar{\rho}, a_{0}, c_{1}, c_{2}, c_{3}$.

(A2) $\forall i \in \mathbb{Z}, t, s \in \mathbb{R}, f_{i}, f_{i, s}^{\prime} \in C(\mathbb{R} \times \mathbb{R}, \mathbb{R})$, and there exist positive constants $a_{1}, a_{2}, a_{3}$, $a_{4}, \underline{\lambda}, \bar{\lambda}>0$ and functions $\beta_{i} \in C^{1}(\mathbb{R}, \mathbb{R})$ such that

$$
\left\{\begin{array}{l}
f_{i}(0, t)=0, \quad\left|f_{i, s}^{\prime}(s, t)\right| \leq a_{1}|s|\left(1+|s|^{p-1}\right), \quad p>1 \\
a_{2} f_{i}(s, t) s \geq G_{i}(s, t) \geq a_{3}|s|^{p+2}-\beta_{i}^{2}(t), \\
G_{i, t}^{\prime}(s, t) \leq a_{4} G_{i}(s, t), \quad 0 \leq a_{4} \leq \frac{\varepsilon}{2 a_{2}} \\
\underline{\lambda} \leq \lambda_{i} \leq \bar{\lambda}<+\infty
\end{array}\right.
$$

(A3) $\forall t \in \mathbb{R}, \beta(t)=\left(\beta_{i}(t)\right)_{i \in \mathbb{Z}}, \beta^{\prime}(t)=\left(\beta_{i}^{\prime}(t)\right)_{i \in \mathbb{Z}}, g(t)=\left(g_{i}(t)\right)_{i \in \mathbb{Z}} \in \mathbf{G}$, where

$$
\mathbf{G}=\left\{g \in C_{b}\left(\mathbb{R}, l_{\rho}^{2}\right): \forall \eta>0, \exists I(\eta) \in \mathbb{N} \text { such that } \sup _{t \in \mathbb{R}} \sum_{|i|>I(\eta)} \rho_{i} g_{i}^{2}(t)<\eta\right\}
$$

and $C_{b}\left(\mathbb{R}, l_{\rho}^{2}\right)$ is the space of all continuous bounded functions from $\mathbb{R}$ into $l_{\rho}^{2}$.

(A4) $c_{3}$ in (A1) satisfies

$$
0 \leq c_{3} \leq \min \left\{\frac{\varepsilon}{2 c_{0} c_{4}}, \frac{\alpha}{2 c_{0} c_{4} c_{2}^{m_{0}}\left(2 m_{0}+1\right)^{2}(\delta+\gamma)}, \frac{2}{c_{0} c_{4}}\right\}
$$

where

$$
\begin{aligned}
& c_{4}=c_{2}^{m_{0}-1}+c_{2}^{m_{0}-2}+\cdots+c_{2}+1, \\
& 0<\varepsilon= \begin{cases}\frac{\alpha \underline{\lambda}}{2 \alpha^{2}+3 \underline{\lambda}}, & \gamma=0, \\
\min \left\{\frac{1}{\gamma}, \frac{\alpha \underline{\lambda}}{2 \alpha^{2}+3 \underline{\lambda}}\right\}, & \gamma \neq 0 .\end{cases}
\end{aligned}
$$

For any $u, v \in l_{\rho}^{2}$, define new inner products on $l_{\rho}^{2}$ by

$$
\begin{aligned}
& (u, v)_{\lambda, \rho}=\sum_{i \in \mathbb{Z}} \rho_{i} \lambda_{i} u_{i} v_{i}, \quad\|u\|_{\lambda, \rho}^{2}=\sum_{i \in \mathbb{Z}} \rho_{i} \lambda_{i} u_{i}^{2}, \\
& (u, v)_{\delta, \lambda, \rho}=\delta(D u, D v)_{\rho}+(u, v)_{\lambda, \rho}, \\
& \|u\|_{\delta, \lambda, \rho}^{2}=\delta\|D u\|_{\rho}^{2}+\|u\|_{\lambda, \rho}^{2}, \quad \delta=1-\varepsilon \gamma \in[0,1],
\end{aligned}
$$

then the norms $\|\cdot\|_{\rho},\|\cdot\|_{\lambda, \rho}$ and $\|\cdot\|_{\delta, \lambda, \rho}$ are equivalent to each other because

$$
\underline{\lambda}\|u\|_{\rho}^{2} \leq\|u\|_{\lambda, \rho}^{2} \leq\|u\|_{\delta, \lambda, \rho}^{2} \leq \frac{\delta \bar{\lambda} c_{0}^{2} c_{2}^{m_{0}}\left(2 m_{0}+1\right)^{2}}{\underline{\lambda}}\|u\|_{\rho}^{2} .
$$

Let $l_{\delta, \lambda, \rho}^{2}=\left(l_{\rho}^{2},(\cdot, \cdot)_{\delta, \lambda, \rho},\|\cdot\|_{\delta, \lambda, \rho}\right), H=l_{\delta, \lambda, \rho}^{2} \times l_{\rho}^{2}$, then $H$ is a separable Hilbert space.

Let $(\Omega, \mathcal{F}, \mathbb{P})$ be defined in Sect. 1 . Define a family of mappings $\left(\theta_{t}\right)_{t \in \mathbb{R}}$ on $\Omega: \theta_{t} \omega(\cdot)=$ $\omega(\cdot+t)-\omega(t)$ for $\omega \in \Omega$, then $\left(\Omega, \mathcal{F}, \mathbb{P},\left(\theta_{t}\right)_{t \in \mathbb{R}}\right)$ is an ergodic metric dynamical system [4]. Let us consider the Ornstein-Uhlenbeck stationary process $z\left(\theta_{t} \omega\right)=-\alpha \int_{-\infty}^{0} e^{\alpha s}\left(\theta_{t} \omega\right)(s) d s$ for $t \in \mathbb{R}$ and $\omega \in \Omega$, which solves the Itô stochastic equation $d z\left(\theta_{t} \omega\right)+\alpha z\left(\theta_{t} \omega\right) d t=$ 
$d W(t, \omega)$, where $W(t, \omega)=\omega(t)$. It is well known that the random variable $|z(\omega)|$ is tempered and there exists $\Omega_{0} \subseteq \Omega$ with $\mathbb{P}\left(\Omega_{0}\right)=1$, such that for every $\omega \in \Omega_{0}, t \mapsto z\left(\theta_{t} \omega\right)$ is continuous in $t$ and

$$
\lim _{t \rightarrow \pm \infty} \frac{\left|z\left(\theta_{t} \omega\right)\right|}{t}=\lim _{t \rightarrow \pm \infty} \frac{\int_{0}^{t} z\left(\theta_{s} \omega\right) d s}{t}=0 .
$$

For convenience, we still write $\Omega_{0}$ as $\Omega$.

Introduce a variable transformation $v=u_{t}+\varepsilon u-c u z\left(\theta_{t} \omega\right)$, then the problem (2) is equivalent to the following random system:

$$
\dot{\varphi}+L \varphi=F\left(\varphi, \theta_{t} \omega\right), \quad \varphi_{\tau}(\omega)=\left(\begin{array}{c}
u_{\tau} \\
v_{\tau}(\omega)
\end{array}\right)=\left(\begin{array}{c}
u_{\tau} \\
u_{1, \tau}+\varepsilon u_{\tau}-c u_{\tau} z\left(\theta_{\tau} \omega\right)
\end{array}\right)
$$

where

$$
\begin{aligned}
& \varphi(t, \tau, \omega)=\left(\begin{array}{l}
u \\
v
\end{array}\right), \quad L=\left(\begin{array}{cc}
\varepsilon & -1 \\
\lambda+A-\varepsilon(\alpha-\varepsilon)-\gamma \varepsilon A \alpha-\varepsilon+\gamma A
\end{array}\right) I, \\
& F\left(\varphi, \theta_{t} \omega\right)=\left(\begin{array}{c}
c u z\left(\theta_{t} \omega\right) \\
{\left[2 \varepsilon c z\left(\theta_{t} \omega\right)-c^{2} z^{2}\left(\theta_{t} \omega\right)\right] u-c z\left(\theta_{t} \omega\right) \nu-} \\
\gamma c z\left(\theta_{t} \omega\right) A u-f(u, t)+g(t)
\end{array}\right) .
\end{aligned}
$$

It follows from assumptions (A1)-(A4) and theory of ordinary differential equations that, for every $\omega \in \Omega, \tau \in \mathbb{R}$ and $\varphi_{\tau}(\omega)=\left(\begin{array}{c}u_{\tau} \\ v_{\tau}(\omega)\end{array}\right) \in H$, the problem (4) has a unique solution $\varphi\left(\cdot, \tau, \omega, \varphi_{\tau}\right) \in C([\tau, \tau+T), H)$ for any $T>0$, where $\varphi\left(\tau, \tau, \omega, \varphi_{\tau}\right)=\varphi_{\tau}$ and $\varphi\left(t, \tau, \omega, \varphi_{\tau}\right)$ is continuous in $\varphi_{\tau} \in H$, which defines a continuous cocycle $\Phi: \mathbb{R}^{+} \times \mathbb{R} \times \Omega \times H \rightarrow H$ by

$$
\left(t, \tau, \omega, \varphi_{\tau}\right) \rightarrow \Phi\left(t, \tau, \omega, \varphi_{\tau}\right)=\Phi(t, \tau, \omega) \varphi_{\tau}=\varphi\left(t+\tau, \tau, \theta_{-\tau} \omega, \varphi_{\tau}\left(\theta_{-\tau} \omega\right)\right)
$$

over $\mathbb{R}$ and $\left(\Omega, \mathcal{F}, \mathbb{P},\left(\theta_{t}\right)_{t \in \mathbb{R}}\right)$ with state space $H$.

\subsection{Random exponential attractor}

In this subsection, we present the definition and existence conditions of a random exponential attractor for the continuous cocycle $\{\Phi(t, \tau, \omega)\}_{t \geq 0, \omega \in \Omega, \tau \in \mathbb{R}}$ in $H$. Let $\mathcal{D}(H)$ be the collection of all the tempered families of nonempty subsets of $H$ [3]. A family $D=$ $\{D(\tau, \omega) \subset H\}_{\tau \in \mathbb{R}, \omega \in \Omega}$ of nonempty subsets of $H$ is said to be tempered with respective to $\left(\theta_{t}\right)_{t \in \mathbb{R}}$ if, for every $\epsilon>0, \tau \in \mathbb{R}$, and a.e. $\omega \in \Omega, \lim _{t \rightarrow \infty} e^{-\epsilon|t|}\left\|D\left(\tau+t, \theta_{t} \omega\right)\right\|_{H}=0$, where $\|D(\tau, \omega)\|_{H}=\sup _{\varphi \in D(\tau, \omega)}\|\varphi\|_{H}$.

Definition 2.1 ([3]) A family $\{\mathcal{A}(\tau, \omega)\}_{\tau \in \mathbb{R}, \omega \in \Omega}$ of subsets of $H$ is called a random exponential attractor in $\mathcal{D}(H)$ for a continuous cocycle $\{\Phi(t, \tau, \omega)\}_{t \geq 0, \tau \in \mathbb{R}, \omega \in \Omega}$ on $H$ over $\mathbb{R}$ and $\left(\Omega, \mathcal{F}, \mathbb{P},\left(\theta_{t}\right)_{t \in \mathbb{R}}\right)$ if, for any $\tau \in \mathbb{R}$ and $\omega \in \Omega$, (i) $\mathcal{A}(\tau, \omega)$ is compact subset in $H$ and measurable in $\omega$; (ii) $\Phi\left(t, \tau-t, \theta_{-t} \omega\right) \mathcal{A}\left(\tau-t, \theta_{-t} \omega\right) \subseteq \mathcal{A}(\tau, \omega)$ for all $t \geq 0$; (iii) there exists a random variable $\zeta_{\omega}(<\infty)$ such that $\sup _{\tau \in \mathbb{R}} \operatorname{dim}_{f} \mathcal{A}(\tau, \omega) \leq \zeta_{\omega}<\infty$, where $\operatorname{dim}_{f} \mathcal{A}(\tau, \omega)=\limsup \sup _{\varepsilon \rightarrow 0^{+}} \frac{\ln N_{\varepsilon}(\mathcal{A}(\tau, \omega))}{-\ln \varepsilon}$ is the fractal dimension of $\mathcal{A}(\tau, \omega)$ and $N_{\varepsilon}(\mathcal{A}(\tau, \omega))$ is the minimal number of balls with radius $\varepsilon$ covering $\mathcal{A}(\tau, \omega)$ in $H$; (iv) there exists a constant $\tilde{a}>0$ such that, for any $D \in \mathcal{D}(H)$, there exist random variables $t_{D}(\tau, \omega) \geq 0$, 
$Q(\tau, \omega, D)>0$ satisfying $\mathrm{d}_{h}\left(\Phi\left(t, \tau-t, \theta_{-t} \omega\right) D\left(\tau-t, \theta_{-t} \omega\right), \mathcal{A}(\tau, \omega)\right) \leq Q(\tau, \omega, D) e^{-\tilde{a} t}$ for all $t \geq t_{D}(\tau, \omega)$, where $d_{h}$ denotes the Hausdorff semidistance between two subsets.

Based on Theorem 2.8 in [13] and Theorems 2.1-2.4 in [3], by making some slightly revision in the proof, we have the following theorem.

Theorem 2.1 Consider the continuous cocycle $\{\Phi(t, \tau, \omega)\}_{t \geq 0, \tau \in \mathbb{R}, \omega \in \Omega}$. Assume that:

(B1) There exist a family of uniformly (with respect to $\tau \in \mathbb{R}$ ) tempered closed measurable set $D_{0}=\left\{D_{0}(\omega)\right\}_{\tau \in \mathbb{R}, \omega \in \Omega} \in \mathcal{D}(H)$ satisfying that, for any $\tau \in \mathbb{R}, \omega \in \Omega$ and $D(\tau, \omega) \in \mathcal{D}(H)$, there exists a $t_{D}(\tau, \omega) \geq 0$ such that $\Phi(t, \tau-t, \omega) D\left(\tau-t, \theta_{-t} \omega\right) \subseteq D_{0}(\omega)$ for all $t \geq t_{D}(\tau, \omega)$. Particularly, there exists a $t_{D_{0}}(\omega) \geq 0$ (independent of $\tau$ ) such that $\Phi(t, \tau-t, \omega) D_{0}\left(\theta_{-t} \omega\right) \subseteq D_{0}(\omega)$ for all $t \geq t_{D_{0}}(\omega)$. For any $\omega \in \Omega, \tau \in \mathbb{R}$, set

$$
\mathcal{X}(\tau, \omega)=\bigcup_{t \geq t_{D_{0}}(\omega)} \Phi\left(t, \tau-t, \theta_{-t} \omega\right) D_{0}\left(\theta_{-t} \omega\right) \subseteq D_{0}(\omega)
$$

(B2) There exist some positive numbers $\hat{\lambda}>0, \hat{\delta}>0, k(\in \mathbb{N})$ (independent of $(\tau, \omega)$, but $\hat{\delta}$, $k$ maybe depend on $\hat{\lambda})$ and random variables $C_{0}(\omega), C_{1}(\omega) \geq 0$ such that for any $\tau \in \mathbb{R}, \omega \in \Omega$ and any $u, v \in \mathcal{X}(\tau, \omega) \subset H$, we have

$$
\begin{aligned}
& \|\Phi(t, \tau, \omega) u-\Phi(t, \tau, \omega) v\|_{H} \leq e^{\int_{0}^{t} C_{0}\left(\theta_{s} \omega\right) d s}\|u-v\|_{H}, \quad \forall t \in\left[0, \frac{8 \ln 2}{\hat{\lambda}}\right] \\
& \sum_{|i|>k} \rho_{i}\left(\Phi\left(\frac{8 \ln 2}{\hat{\lambda}}, \tau, \omega\right) u-\Phi\left(\frac{8 \ln 2}{\hat{\lambda}}, \tau, \omega\right) v\right)_{i}^{2} \\
& \leq\left(e^{-8 \ln 2+\int_{0}^{\frac{8 \ln 2}{\hat{\lambda}}}} C_{1}\left(\theta_{s} \omega\right) d s+\frac{\hat{\delta}}{2} e^{\int_{0}^{\frac{8 \ln 2}{\hat{\lambda}}} C_{0}\left(\theta_{s} \omega\right) d s}\right)^{2}\|u-v\|_{H}^{2}
\end{aligned}
$$

and

$$
\left\{\begin{array}{l}
0 \leq \mathbf{E}\left[C_{1}(\omega)\right] \leq \frac{\hat{\lambda}}{16}, \quad 0 \leq \mathbf{E}\left[C_{0}^{2}(\omega)\right]<\infty, \\
0<\hat{\delta} \leq \min \left\{\frac{1}{16}, e^{-\frac{128 \ln ^{2} 2}{\hat{\lambda}^{2} \ln \frac{3}{2}}\left(\mathbf{E}\left[C_{0}^{2}(\omega)\right]+\hat{\lambda} \mathbf{E}\left[C_{0}(\omega)\right]\right)}\right\}
\end{array}\right.
$$

where $\mathbf{E}$ denotes the expectation.

(B3)

$$
\left\{\begin{array}{l}
\lim _{t \rightarrow 0^{+}} \sup _{u \in \mathcal{X}(\tau, \omega)}\|\Phi(t, \tau, \omega) u-u\|_{H}=0 \\
\lim _{t \rightarrow 0^{+}} \sup _{u \in \mathcal{X}\left(\tau-t, \theta_{-t} \omega\right)}\left\|\Phi\left(0, \tau-t, \theta_{-t} \omega\right) u-u\right\|_{H}=0
\end{array}\right.
$$

Then $\{\Phi(t, \tau, \omega)\}_{t \geq 0, \tau \in \mathbb{R}, \omega \in \Omega}$ has a random exponential attractor $\{\mathcal{A}(\tau, \omega)\}_{\tau \in \mathbb{R}, \omega \in \Omega}$ such that, for every $\tau \in \mathbb{R}, \omega \in \Omega$ and $t \geq 0$,

(i) $\mathcal{A}(\tau, \omega)(\subseteq\{\overline{\mathcal{X}(\tau, \omega)}\})$ is a compact set of $H$ and measurable in $\omega$;

(ii) $\Phi(t, \tau, \omega) \mathcal{A}(\tau, \omega) \subseteq \mathcal{A}\left(\tau+t, \theta_{t} \omega\right)$;

(iii) $\operatorname{dim}_{f} \mathcal{A}(\tau, \omega) \leq \frac{4(2 k+1) \ln \left(\frac{2 \sqrt{2(2 k+1)}}{\hat{\delta}}+1\right)}{\ln \frac{4}{3}}<\infty$ 
(iv) for every set $D \in \mathcal{D}(H)$, there exist a random variable $T_{\omega, D} \geq 0$ and a tempered random variable $b_{\omega, D}>0$ such that

$$
\mathrm{d}_{h}\left(\Phi(t, \tau, \omega) D(\tau, \omega), \mathcal{A}\left(t+\tau, \theta_{t} \omega\right)\right) \leq b_{\omega, D} e^{-\frac{\hat{\lambda} \ln \frac{4}{3}}{32 \ln 2} t}, \quad t \geq T_{\omega, D}
$$

(v) for any $\tau \in \mathbb{R}, \omega \in \Omega, \lim _{t \rightarrow 0} \mathrm{~d}_{h}\left(\mathcal{A}\left(\tau+t, \theta_{t} \omega\right), \mathcal{A}(\tau, \omega)\right)=0$.

Remark 2.1 The condition (B2) in Theorem 2.1 above is different from the corresponding condition in the well-known publication [3].

In the following, we will prove the existence of a random exponential attractor for $\Phi$ based on Theorem 2.1.

\subsection{Estimations of bound and tail of solutions}

Write $\|g\|_{\rho}=\sup _{r \in \mathbb{R}}\|g(r)\|_{\rho},\|\beta\|_{\rho}=\sup _{r \in \mathbb{R}}\|\beta(r)\|_{\rho}$ and

$$
M_{0}^{2}(\omega)=c_{5} K_{0}(\omega), \quad K_{0}(\omega)=\int_{-\infty}^{0} e^{\mu s+a_{7} \int_{s}^{0}\left(|c|\left|z\left(\theta_{l} \omega\right)\right|+|c|^{2}\left|z\left(\theta_{l} \omega\right)\right|^{2}\right) d l} d s
$$

where

$$
\left\{\begin{array}{l}
a_{7}=\max \left\{\frac{2 a_{6}}{\underline{\lambda}}+\frac{2 \varepsilon}{\sqrt{\underline{\lambda}}}+2, \frac{1}{\sqrt{\lambda}}+\frac{8 a_{5}^{2}}{\alpha \underline{\underline{\lambda}}}, \frac{a_{1}+1}{a_{3}}\right\}, \\
a_{5}=|\gamma| c_{0}^{2} c_{2}^{m_{0}}\left(2 m_{0}+1\right)^{2}, \quad a_{6}=\frac{p-1}{p^{\frac{p}{p-1}}}, \\
c_{5}=\frac{8}{\alpha}\|g\|_{\rho}^{2}+2\|\beta\|_{\rho}^{2}, \quad \mu=\min \left\{\varepsilon, \frac{\varepsilon}{2 a_{2}}, 1\right\} .
\end{array}\right.
$$

In the following part of this section, we always assume that conditions (A1)-(A4) and

$$
a_{7}\left(\frac{|c|}{\sqrt{\pi \alpha}}+\frac{|c|^{2}}{2 \alpha}\right) \leq \frac{\mu}{2}
$$

hold.

Lemma 2.1 For every $\tau \in \mathbb{R}, \omega \in \Omega$ and $D \in \mathcal{D}(H)$, there exist $T_{D}(\tau, \omega) \geq 0$ and a tempered random variable $M_{0}(\omega)$ (independent of $\tau$ ), such that the solution $\varphi(r, \tau-$ $\left.t, \theta_{-\tau} \omega, \varphi_{\tau-t}\left(\theta_{-\tau} \omega\right)\right) \in H(r \geq \tau-t)$ of (4) with $\varphi_{\tau-t}\left(\theta_{-\tau} \omega\right) \in D\left(\tau-t, \theta_{-t} \omega\right)$ satisfies

$$
\varphi\left(\tau, \tau-t, \theta_{-\tau} \omega, \varphi_{\tau-t}\left(\theta_{-\tau} \omega\right)\right) \leq M_{0}(\omega), \quad \forall t \geq T(\tau, \omega, D) .
$$

Proof From (3) and (8), it is easy to see that $M_{0}(\omega)$ is a tempered random variable. Taking the inner product of (4) with $\varphi(r)=\varphi\left(r, \tau-t, \theta_{-\tau} \omega, \varphi_{\tau-t}\left(\theta_{-\tau} \omega\right)\right)$ in $H$, we have

$$
\frac{1}{2} \frac{d}{d t}\|\varphi\|_{H}^{2}+(L(\varphi), \varphi)_{H}=\left(f\left(\varphi, \theta_{r-\tau} \omega\right), \varphi\right)_{H}
$$

The second term of (9) is

$$
(L \varphi, \varphi)_{H}=\varepsilon\|u\|_{\delta, \lambda, \rho}^{2}-\delta(D u, D v)_{\rho}-(v, u)_{\lambda, \rho}+\lambda(u, v)_{\rho}+(1-\gamma \varepsilon)(A u, v)_{\rho}
$$




$$
-\varepsilon(\alpha-\varepsilon)(u, v)_{\rho}+(\alpha-\varepsilon)(\nu, v)_{\rho}+\gamma(A v, v)_{\rho} .
$$

From [14], we have

$$
\begin{aligned}
& (A u, v)_{\rho} \geq(D u, D v)_{\rho}-\frac{1}{2} c_{0} c_{3} c_{4}\|D u\|_{\rho}^{2}-\frac{1}{2} c_{0} c_{2}^{m_{0}} c_{3} c_{4}\left(2 m_{0}+1\right)^{2}\|v\|_{\rho}^{2}, \\
& (A v, v)_{\rho} \geq\left(1-\frac{1}{2} c_{0} c_{3} c_{4}\right)\|D v\|_{\rho}^{2}-\frac{1}{2} c_{0} c_{2}^{m_{0}} c_{3} c_{4}\left(2 m_{0}+1\right)^{2}\|v\|_{\rho}^{2} .
\end{aligned}
$$

By (A4), (10)-(12), we have

$$
\begin{aligned}
(L \varphi, \varphi)_{H} \geq & \frac{3 \varepsilon}{4}\|u\|_{\delta, \lambda, \rho}^{2}-\varepsilon(\alpha-\varepsilon)(u, v)_{\rho}+\left(\frac{3 \alpha}{4}-\varepsilon\right)\|v\|_{\rho}^{2} \\
& +\left(\frac{\delta \varepsilon}{4}-\frac{\delta}{2} c_{0} c_{3} c_{4}\right)\|D u\|_{\rho}^{2}+\gamma\left(1-\frac{1}{2} c_{0} c_{3} c_{4}\right)\|D v\|_{\rho}^{2} \\
& +\left(\frac{\alpha}{4}-\frac{\delta+\gamma}{2} c_{0} c_{2}^{m_{0}} c_{3} c_{4}\left(2 m_{0}+1\right)^{2}\right)\|v\|_{\rho}^{2} \\
\geq & \frac{\varepsilon}{2}\|u\|_{\delta, \lambda, \rho}^{2}+\frac{\varepsilon}{2}\|v\|_{\rho}^{2}+\frac{\alpha}{4}\|v\|_{\rho}^{2}+\frac{\varepsilon}{4}\|u\|_{\delta, \lambda, \rho}^{2}+\left(\frac{3 \alpha}{4}-\varepsilon\right)\|v\|_{\rho}^{2} \\
& +\left(\frac{\alpha}{2}-\frac{3 \varepsilon}{2}\right)\|v\|_{\rho}^{2}-\frac{\varepsilon \alpha}{\sqrt{\lambda}}\|u\|_{\delta, \lambda, \rho}\|v\|_{\rho} .
\end{aligned}
$$

From (A4), $4 \frac{\varepsilon}{4}\left(\frac{\alpha}{2}-\frac{3 \varepsilon}{2}\right) \geq \frac{\varepsilon^{2} \alpha^{2}}{\underline{\lambda}}$, we have

$$
(L \varphi, \varphi)_{H} \geq \frac{\varepsilon}{2}\|\varphi(r)\|_{H}^{2}+\frac{\alpha}{4}\|v\|_{\rho}^{2} .
$$

The right-hand side of (9) is

$$
\left(f\left(\varphi, \theta_{r-\tau} \omega\right), \varphi\right)_{H}=(c z u, u)_{\delta, \lambda, \rho}+\left(-c z v+2 \varepsilon c z u-c^{2} z^{2} u+g(r)-\gamma c A z u-f(u, r), v\right)_{\rho}
$$

For each term of (13), we have

$$
\begin{aligned}
&(c z u, u)_{\delta, \lambda, \rho}-(c z v, v)_{\rho} \leq|c||z| \mid \varphi(r)\left\|_{H}^{2}, \quad(g, v)_{\rho} \leq \frac{4}{\alpha}\right\| g\left\|_{\rho}^{2}+\frac{\alpha}{8}\right\| v \|_{\rho}^{2}, \\
&(2 \varepsilon c z u, v)_{\rho}-\left(c^{2} z^{2} u, v\right)_{\rho} \leq\left(\frac{|c||\varepsilon||z|}{\sqrt{\underline{\lambda}}}+\frac{|c|^{2}|z|^{2}}{2 \sqrt{\lambda}}\right)\|\varphi(r)\|_{H}^{2}, \\
&-(\gamma c A z u, v)_{\rho} \leq \frac{4|\gamma c z|^{2}\|A u\|_{\rho}^{2}}{\alpha}+\frac{\alpha}{8}\|v\|_{\rho}^{2} \\
& \leq \frac{4|\gamma c z|^{2} c_{0}^{4} c_{2}^{2 m_{0}}\left(2 m_{0}+1\right)^{4}}{\alpha \underline{\lambda}}\|u\|_{\lambda, \rho}^{2}+\frac{\alpha}{8}\|v\|_{\rho}^{2} \\
& \leq \frac{4|c z|^{2} a_{5}^{2}}{\alpha \underline{\lambda}}\|\varphi\|_{H}^{2}+\frac{\alpha}{8}\|v\|_{\rho}^{2}, \\
&-(f(u, r), v)_{\rho}=-(f(u, r), \dot{u})_{\rho}-\varepsilon(f(u, r), u)_{\rho}+c z(f(u, r), u)_{\rho} .
\end{aligned}
$$


By (A2) we have

$$
\begin{aligned}
(f(u, r), \dot{u})_{\rho} & =\sum_{i \in \mathbb{Z}} \rho_{i} f_{i}\left(u_{i}, r\right) \dot{u}_{i}=\frac{d}{d t}\left(\sum_{i \in \mathbb{Z}} \rho_{i} G_{i}\left(u_{i}, r\right)\right)-\sum_{i \in \mathbb{Z}} \rho_{i} G_{i, r}^{\prime}\left(u_{i}, r\right) \\
& \geq \frac{d}{d t} \bar{G}(u, r)-a_{4} \bar{G}(u, r) \geq \frac{d}{d t} \bar{G}(u, r)-\frac{\varepsilon}{2 a_{2}} \bar{G}(u, r), \\
\varepsilon(f(u, r), u)_{\rho} & =\varepsilon \sum_{i \in \mathbb{Z}} \rho_{i} f_{i}\left(u_{i}, r\right) u_{i} \geq \frac{\varepsilon}{a_{2}} \sum_{i \in \mathbb{Z}} \rho_{i} G_{i}\left(u_{i}, r\right)=\frac{\varepsilon}{a_{2}} \bar{G}(u, r) .
\end{aligned}
$$

By the Young inequality, we obtain

$$
a_{1}|s| \leq|s|^{p}+a_{1}^{\frac{p}{p-1}} \times \frac{p-1}{p^{\frac{p}{p-1}}}=|s|^{p}+a_{6}, \quad p>1 .
$$

By (A4) and (20),

$$
\left|f_{i}^{\prime}(s, r)\right| \leq a_{1}|s|+a_{1}|s|^{p} \leq a_{6}+\left(1+a_{1}\right)|s|^{p},
$$

from (21), we get

$$
\left|f_{i}(s, r)\right| \leq a_{6}|s|+\left(1+a_{1}\right)|s|^{p+1} .
$$

From (22), we have

$$
\begin{aligned}
c z(f(u, r), u)_{\rho}= & c z \sum_{i \in \mathbb{Z}} \rho_{i} f_{i}\left(u_{i}, r\right) u_{i} \leq|c||z|\left|\sum_{i \in \mathbb{Z}} \rho_{i} f_{i}\left(u_{i}, r\right) u_{i}\right| \\
\leq & a_{6}|c||z|\|u\|_{\rho}^{2}+\frac{\left(a_{1}+1\right)}{a_{3}}|c||z| \sum_{i \in \mathbb{Z}} \rho_{i}\left(G_{i}\left(u_{i}, r\right)+\beta_{i}^{2}(r)\right) \\
\leq & a_{6}|c||z|\|u\|_{\rho}^{2}+\frac{\left(a_{1}+1\right)}{a_{3}}|c||z| \bar{G}(u, r) \\
& +\left.\frac{\left(a_{1}+1\right)}{a_{3}}|c\|z\|| \beta\right|_{\rho} ^{2} .
\end{aligned}
$$

Thus, by all the above inequalities, we have

$$
\begin{aligned}
\frac{d}{d t}\left[\|\varphi(r)\|_{H}^{2}+2 \bar{G}(u, r)+2\|\beta\|_{\rho}^{2}\right]+\mu\left[\|\varphi(r)\|_{H}^{2}+2 \bar{G}(u, r)+2\|\beta\|_{\rho}^{2}\right] \\
\leq\left(2|c||z|+\frac{2|c||\varepsilon||z|}{\sqrt{\underline{\lambda}}}+\frac{|c|^{2}|z|^{2}}{\sqrt{\bar{\lambda}}}+\frac{8|c z|^{2} \mid a_{5}^{2}}{\alpha \underline{\lambda}}+\frac{2 a_{6}|c||z|}{\underline{\lambda}}\right)\|\varphi(r)\|_{H}^{2} \\
\quad+\frac{2\left(a_{1}+1\right)}{a_{3}}|c||z| \bar{G}(u, r)+\frac{2\left(a_{1}+1\right)}{a_{3}}|c||z| \beta\left\|_{\rho}^{2}+\frac{8}{\alpha}\right\| g\left\|_{\rho}^{2}+2\right\| \beta \|_{\rho}^{2} .
\end{aligned}
$$

Then, for $r \geq \tau-t$, we have

$$
\frac{d}{d t} y(r) \leq\left(-\mu+a_{7}\left(|c|\left|z\left(\theta_{r-\tau} \omega\right)\right|+|c|^{2}\left|z\left(\theta_{r-\tau} \omega\right)\right|^{2}\right) y(r)+c_{5}\right.
$$


where

$$
y(r)=\|\varphi(r)\|_{H}^{2}+2 \bar{G}(u, r)+2|\beta|_{\rho}^{2} \geq\|\varphi(r)\|_{H}^{2} .
$$

By the Gronwall inequality applied to $(24)$ on $[\tau-t, r]$, we obtain

$$
\begin{aligned}
\|\varphi(r)\|_{H}^{2} \leq & e^{\int_{\tau-t}^{r}\left(-\mu+a_{7}\left(|c|\left|z\left(\theta_{l-\tau} \omega\right)\right|+|c|^{2}\left|z\left(\theta_{l-\tau} \omega\right)\right|^{2}\right) d l\right.} y(\tau-t) \\
& +c_{5} \int_{\tau-t}^{r} e^{\int_{s}^{r}\left(-\mu+a_{7}\left(|c|\left|z\left(\theta_{l-\tau} \omega\right)\right|+|c|^{2}\left|z\left(\theta_{l-\tau} \omega\right)\right|^{2}\right) d l\right.} d s
\end{aligned}
$$

and let $r=\tau$, it follows that

$$
\|\varphi(\tau)\|_{H}^{2} \leq e^{\int_{-t}^{0}\left(-\mu+a_{7}\left(|c|\left|z\left(\theta_{l} \omega\right)\right|+|c|^{2}\left|z\left(\theta_{l} \omega\right)\right|^{2}\right) d l\right.} y(\tau-t)+\frac{1}{2} M_{0}^{2}(\omega) .
$$

By $\varphi_{\tau-t}\left(\theta_{-\tau} \omega\right) \in D\left(\tau-t, \theta_{-t} \omega\right) \in \mathcal{D}(H)$ and (8), we have

$$
\lim _{t \rightarrow+\infty} e_{-t}^{\int_{-t}^{0}\left(-\mu+a_{7}\left(|c|\left|z\left(\theta_{l} \omega\right)\right|+|c|^{2}\left|z\left(\theta_{l} \omega\right)\right|^{2}\right) d l\right.} y(\tau-t)=0
$$

Thus, the proof is completed.

Let

$$
D_{0}=\left\{D_{0}(\omega)=\left\{\varphi \in H:\|\varphi\|_{H} \leq M_{0}(\omega)\right\}\right\} \in D(H),
$$

then $D_{0}$ is a tempered family. By Lemma 2.1, there exists $T_{D_{0}}(\omega)>0$ (independent of $\tau$ ) such that, for any $\omega \in \Omega, \tau \in R$,

$$
\varphi\left(r, \tau-t, \theta_{-\tau} \omega, D_{0}\left(\theta_{-t} \omega\right)\right) \in D_{0}(\omega), \quad \forall r \geq \tau-t, t \geq T_{D_{0}}(\omega) \geq 0 .
$$

Choosing a smooth increasing function $\eta \in C^{1}\left(\mathbb{R}^{+}, \mathbb{R}\right)$ satisfies

$$
\begin{cases}\eta(s)=0, & s \in[0,1) ; \\ 0 \leq \eta(s) \leq 1, & s \in[1,2) ; \\ \eta(s)=1, & s \in[2,+\infty) ; \\ \left|\eta^{\prime}(s)\right| \leq C_{0}, & \forall s \in \mathbb{R}^{+}, C_{0}>0 .\end{cases}
$$

Lemma 2.2 For every $\tau \in \mathbb{R}, t \geq 0, \omega \in \Omega, I \in \mathbb{N}$ and $v>0$, there exists $T_{\nu}(\omega)>0$ (independent of $\tau)$ such that the solution $\varphi(r)=\varphi\left(r, \tau-t, \theta_{-\tau} \omega, \varphi_{\tau-t}\left(\theta_{-\tau} \omega\right)(r \geq \tau-t)\right.$ of (4) with $\varphi_{\tau-t}\left(\theta_{-\tau} \omega\right) \in D_{0}\left(\theta_{-t} \omega\right)$ satisfies

$$
\sum_{|i| \geq 2 I}\left|\varphi_{i}\left(\tau, \tau-t, \theta_{-\tau} \omega, \varphi_{\tau-t}\right)\right|_{H}^{2} \leq v+\left(\frac{c_{6}}{I}+\gamma_{I}\right) K_{2}(\omega), \quad t>T_{\nu}(\omega), I \in \mathbb{N}
$$


where

$$
\left\{\begin{array}{l}
\left|\varphi_{i}\right|_{H}^{2}=\rho_{i}\left[\delta(D u)_{i}^{2}+\lambda_{i} u_{i}^{2}+v_{i}^{2}\right], \quad c_{6}=\frac{c_{5}}{2} ; \\
\gamma_{I}=\sup _{r \in \mathbb{R}} \sum_{i \geq I} \rho_{i}\left(\frac{8}{\alpha} g_{i}^{2}(r)+4 \beta_{i}^{2}(r)+4 \beta_{i}^{\prime 2}(r)\right) ; \\
K_{2}(\omega)=K_{0}(\omega)\left(1+K_{1}(\omega)\right) ; \\
K_{1}(\omega)=\int_{-\infty}^{0} \xi_{1}\left(\theta_{s}(\omega) e^{\mu s+a_{7} \int_{s}^{0}\left(|c|||\left(\left.\theta(\theta)|+| c\right|^{2}\left|z\left(\theta_{l} \omega\right)\right|^{2}\right) d l\right.} d s ;\right. \\
\xi_{1}(\omega)=2 c_{7}(\underline{\lambda}+\varepsilon+2|c z(\omega)|)+2 c_{8}\left(\delta+\gamma_{0}\right) ; \\
c_{7}=\frac{\delta m_{0} C_{0} c_{0}^{2}\left(2 m_{0}+1\right)^{2} c_{2}^{m_{0}}}{\underline{\lambda}}, \quad c_{8}=m_{0} C_{0} c_{0} c_{2}^{\frac{m_{0}}{2}}\left(1+\left(2 m_{0}+1\right)^{2}\right) .
\end{array}\right.
$$

Proof Let $I \in \mathbb{N}$ be a suitable large integer, and set $x_{i}=\eta\left(\frac{|i|}{I}\right) u_{i}, y_{i}=\eta\left(\frac{|i|}{I}\right) v_{i}, w=(x, y)=$ $\left(x_{i}, y_{i}\right)_{i \in \mathbb{Z}}^{T}$. Taking the inner product $(\cdot, \cdot)_{H}$ of (4) with $w$, we have

$$
(\dot{\varphi}, w)_{H}+(L \varphi, w)_{H}=(F(\varphi), w)_{H} .
$$

By some computations, it follows that

$$
\begin{aligned}
& (\dot{\varphi}, w)_{H} \geq \frac{1}{2} \frac{d}{d t} \sum_{i \in \mathbb{Z}} \eta\left(\frac{|i|}{I}\right)\left|\varphi_{i}\right|_{H}^{2}-\frac{c_{7}}{I}\left(\underline{\lambda}+\varepsilon+\left|c z\left(\theta_{r-\tau} \omega\right)\right|\right)\|\varphi\|_{H}^{2}, \\
& (L \varphi, w)_{H} \geq \sum_{i \in \mathbb{Z}} \eta\left(\frac{|i|}{I}\right)\left(\frac{\varepsilon}{2}\left|\varphi_{i}\right|_{H}^{2}+\frac{\alpha}{4} \rho_{i} v_{i}^{2}\right)-\frac{c_{8}(\delta+\gamma)}{I}\|\varphi\|_{H}^{2}, \\
& \left(c z\left(\theta_{r-\tau} \omega\right) u, x\right)_{\delta, \lambda, \rho}-\left(c z\left(\theta_{r-\tau} \omega\right) v, y\right)_{\rho} \\
& \leq|c|\left|z\left(\theta_{r-\tau} \omega\right)\right|\left(\sum_{i \in \mathbb{Z}} \eta\left(\frac{|i|}{I}\right)\left|\varphi_{i}\right|_{H}^{2}+\frac{c_{7}}{I}\|\varphi\|_{H}^{2}\right), \\
& \left(2 \varepsilon c z\left(\theta_{r-\tau} \omega\right) u, y\right)_{\rho} \leq \frac{\varepsilon|c|\left|z\left(\theta_{r-\tau} \omega\right)\right|}{\sqrt{\underline{\lambda}}} \sum_{i \in \mathbb{Z}} \eta\left(\frac{|i|}{I}\right)\left|\varphi_{i}\right|_{H}^{2}, \\
& \left(-c^{2} z^{2}\left(\theta_{r-\tau} \omega\right) u, y\right)_{\rho} \leq \frac{c^{2} z^{2}\left(\theta_{r-\tau} \omega\right)}{2 \sqrt{\lambda}} \sum_{i \in \mathbb{Z}} \eta\left(\frac{|i|}{I}\right)\left|\varphi_{i}\right|_{H}^{2}, \\
& \left(-\gamma c A z\left(\theta_{r-\tau} \omega\right) u, y\right)_{\rho} \leq \frac{4|c z|^{2} a_{5}^{2}}{\underline{\lambda} \alpha} \sum_{i \in \mathbb{Z}} \eta\left(\frac{|i|}{I}\right)\left|\varphi_{i}\right|_{H}^{2} \\
& +\frac{\alpha}{8} \sum_{i \in \mathbb{Z}} \eta\left(\frac{|i|}{I}\right) \rho_{i} v_{i}^{2} \\
& (g, y)_{\rho} \leq \frac{4}{\alpha} \sum_{i \in \mathbb{Z}} \eta\left(\frac{|i|}{I}\right) \rho_{i} g_{i}^{2}+\frac{\alpha}{8} \sum_{i \in \mathbb{Z}} \eta\left(\frac{|i|}{I}\right) \rho_{i} v_{i}^{2}, \\
& (f(u, r), y)_{\rho} \\
& \geq \frac{d}{d t} \sum_{i \in \mathbb{Z}} \eta\left(\frac{|i|}{I}\right) \rho_{i} G_{i}\left(u_{i}, r\right)+\frac{\varepsilon}{2 a_{2}} \sum_{i \in \mathbb{Z}} \eta\left(\frac{|i|}{I}\right) \rho_{i} G_{i}\left(u_{i}, r\right) \\
& -a_{6}|c||z| \sum_{i \in \mathbb{Z}} \eta\left(\frac{|i|}{I}\right) \rho_{i} u_{i}^{2}-\frac{a_{1}+1}{a_{3}}|c||z| \sum_{i \in \mathbb{Z}} \eta\left(\frac{|i|}{I}\right) \rho_{i} G_{i}\left(u_{i}, r\right) \\
& -\frac{a_{1}+1}{a_{3}}|c||z| \sum_{i \in \mathbb{Z}} \eta\left(\frac{|i|}{I}\right) \rho_{i} \beta_{i}^{2}(r) .
\end{aligned}
$$


Thus, by putting (30)-(37) into (29), we obtain, for $r \geq \tau-t$,

$$
\begin{aligned}
& \frac{d}{d t} \sum_{i \in \mathbb{Z}} \eta\left(\frac{|i|}{I}\right)\left[\left|\varphi_{i}(r)\right|_{H}^{2}+2 \rho_{i} G_{i}\left(u_{i}, r\right)+2 \rho_{i} \beta_{i}^{2}(r)\right] \\
& \leq\left(-\mu+a_{7}\left(|c|\left|z\left(\theta_{r-\tau} \omega\right)\right|+|c|^{2}\left|z\left(\theta_{r-\tau} \omega\right)\right|^{2}\right)\right. \\
& \quad \times \sum_{i \in \mathbb{Z}} \eta\left(\frac{|i|}{I}\right)\left[\left|\varphi_{i}(r)\right|_{H}^{2}+2 \rho_{i} G_{i}\left(u_{i}, r\right)+2 \rho_{i} \beta_{i}^{2}(r)\right] \\
& \quad+\frac{\xi_{1}\left(\theta_{r-\tau} \omega\right)}{I}\|\varphi(r)\|_{H}^{2}+\sum_{i \in \mathbb{Z}} \eta\left(\frac{|i|}{I}\right) \rho_{i}\left[\frac{8}{\alpha} g_{i}^{2}(r)+4 \beta_{i}^{2}(r)+4 \beta_{i}^{\prime 2}(r)\right]
\end{aligned}
$$

By applying the Gronwall inequality to (38) on $[\tau-t, \tau]$, we have

$$
\begin{aligned}
\sum_{i \in \mathbb{Z}} & \eta\left(\frac{|i|}{I}\right)\left[\left|\varphi_{i}(\tau)\right|_{H}^{2}+2 \rho_{i} G_{i}\left(u_{i, \tau}, \tau\right)+2 \rho_{i} \beta_{i}^{2}(\tau)\right] \\
\leq & {\left[\left\|\varphi_{\tau-t}\right\|_{H}^{2}+2 G\left(u_{\tau-t}, \tau-t\right)+2\|\beta(\tau-t)\|_{\rho}^{2}\right] } \\
& \times e^{\int_{-t}^{0}\left[-\mu+a_{7}\left(|c|\left|z\left(\theta_{l} \omega\right)\right|+|c|^{2}\left|z\left(\theta_{l} \omega\right)\right|^{2}\right)\right] d l} \\
& +\sup _{r \in \mathbb{R}} \sum_{i \geq I} \rho_{i}\left(\frac{8}{\alpha} g_{i}^{2}(r)+4 \beta_{i}^{2}(r)+4 \beta_{i}^{\prime 2}(r)\right) \\
& \times \int_{-t}^{0} e^{\int_{s}^{0}\left[-\mu+a_{7}\left(|c|\left|z\left(\theta_{l} \omega\right)\right|+|c|^{2}\left|z\left(\theta_{l} \omega\right)\right|^{2}\right)\right] d l} d s \\
& +\frac{1}{I} \int_{\tau-t}^{\tau} \xi_{1}\left(\theta_{s-\tau} \omega\right)\|\varphi(s)\|_{H}^{2} \\
& \times e^{\int_{s}^{\tau}\left[-\mu+a_{7}\left(|c|\left|z\left(\theta_{l-\tau} \omega\right)\right|+|c|^{2}\left|z\left(\theta_{l-\tau} \omega\right)\right|^{2}\right)\right] d l} d s .
\end{aligned}
$$

Since $\xi_{1}(\omega)$ is tempered and $\xi_{1}\left(\theta_{t} \omega\right)$ is continuous in $t$, by Proposition 4.3.3 of [15], for the fixed number $\mu>0$, there exists a tempered random variable $\sigma(\omega)$ such that $\xi_{1}\left(\theta_{t} \omega\right) \leq$ $\sigma(\omega) e^{\frac{\mu}{3}|t|}$ for $t \in \mathbb{R}$. Thus, by (25),

$$
\begin{aligned}
& \int_{\tau-t}^{\tau} \xi_{1}\left(\theta_{s-\tau} \omega\right)\|\varphi(s)\|_{H}^{2} e^{\int_{s}^{\tau}\left[-\mu+a_{7}\left(|c|\left|z\left(\theta_{l-\tau} \omega\right)\right|+|c|^{2}\left|z\left(\theta_{l-\tau} \omega\right)\right|^{2}\right)\right] d l} d s \\
& \leq y(\tau-t) e^{\int_{\tau-t}^{\tau}\left(-\mu+a_{7}\left(|c|\left|z\left(\theta_{l-\tau} \omega\right)\right|+|c|^{2}\left|z\left(\theta_{l-\tau} \omega\right)\right|^{2}\right) d l\right.} \int_{\tau-t}^{\tau} \xi_{1}\left(\theta_{s-\tau} \omega\right) d s \\
& \quad+\frac{1}{2} M_{0}^{2}(\omega) \int_{\tau-t}^{\tau} \xi_{1}\left(\theta_{s-\tau} \omega\right) e^{\int_{s}^{\tau}\left[-\mu+a_{7}\left(|c|\left|z\left(\theta_{l-\tau} \omega\right)\right|+|c|^{2}\left|z\left(\theta_{l-\tau} \omega\right)\right|^{2}\right)\right] d l} d s .
\end{aligned}
$$

From (A4) and (22)

$$
\begin{aligned}
y(\tau & \left.-t, \tau-t, \theta_{-t} \omega, \varphi_{\tau-t}\right) \\
& =\left\|\varphi_{\tau-t}\left(\theta_{-\tau} \omega\right)\right\|_{H}^{2}+2 \sum_{i \in \mathbb{Z}} \rho_{i} f_{i}\left(u_{i, \tau-t}, \tau-t\right) u_{i, \tau-t}+2|\beta|_{\rho}^{2} \\
& \leq\left\|\varphi_{\tau-t}\left(\theta_{-t} \omega\right)\right\|_{H}^{2}+2 a_{2}\left(a_{6}\left\|u_{\tau-t}\right\|_{\rho}^{2}+\left(1+a_{1}\right)\left\|u_{\tau-t}\right\|_{\rho}^{p+2}\right)+2|\beta|_{\rho}^{2},
\end{aligned}
$$


thus, (40) can be written as

$$
\begin{aligned}
\int_{\tau-t}^{\tau} & \xi_{1}\left(\theta_{s-\tau} \omega\right) y(s) e^{\int_{s}^{\tau}\left[-\mu+a_{7}\left(|c|\left|z\left(\theta_{l-\tau} \omega\right)\right|+|c|^{2}\left|z\left(\theta_{l-\tau} \omega\right)\right|^{2}\right)\right] d l} d s \\
\leq & \frac{\mu c_{9}}{3} \sigma(\omega)\left(M_{0}^{2}\left(\theta_{-t} \omega\right)+M_{0}^{p+2}\left(\theta_{-t} \omega\right)+1\right) e^{-\frac{2 \mu}{3} t+a_{7} \int_{-t}^{0}\left(|c|\left|z\left(\theta_{l} \omega\right)\right|+|c|^{2}\left|z\left(\theta_{l} \omega\right)\right|^{2}\right) d l} \\
& \quad+\frac{1}{2} M_{0}^{2}(\omega) \int_{-\infty}^{0} \xi_{1}\left(\theta_{s} \omega\right) e^{\int_{s}^{0}\left[-\mu+a_{7}\left(|c|\left|z\left(\theta_{l} \omega\right)\right|+|c|^{2}\left|z\left(\theta_{l} \omega\right)\right|^{2}\right)\right] d l} d s
\end{aligned}
$$

where $c_{9}=\max \left\{1+\frac{2 a_{2} a_{6}}{\underline{\lambda}}, \frac{2 a_{2}\left(a_{1}+1\right)}{\underline{\lambda}^{\frac{p}{2}+1}}, 2\|\beta\|_{\rho}^{2}\right\}$, and

$$
\begin{aligned}
& \int_{-\infty}^{0} \xi_{1}\left(\theta_{s} \omega\right) e^{\int_{s}^{0}\left[-\mu+a_{7}\left(|c|\left|z\left(\theta_{l} \omega\right)\right|+|c|^{2}\left|z\left(\theta_{l} \omega\right)\right|^{2}\right)\right] d l} d s \\
& \quad \leq \sigma(\omega) \int_{-\infty}^{0} e^{\int_{s}^{0}\left[-\frac{2 \mu}{3}+a_{7}\left(|c|\left|z\left(\theta_{l} \omega\right)\right|+|c|^{2}\left|z\left(\theta_{l} \omega\right)\right|^{2}\right)\right] d l} d s<\infty .
\end{aligned}
$$

Thus, by (39) and $I \geq 1$, we have

$$
\begin{aligned}
& \sum_{i \in \mathbb{Z}} \eta\left(\frac{|i|}{I}\right)\left[\left|\varphi_{i}(\tau)\right|_{H}^{2}+2 \rho_{i} G_{i}\left(u_{i, \tau}, \tau\right)+2 \rho_{i} \beta_{i}^{2}(\tau)\right] \\
& \leq c_{9}\left(M_{0}^{2}\left(\theta_{-t} \omega\right)+M_{0}^{p+2}\left(\theta_{-t} \omega\right)+1\right) e^{\int_{-t}^{0}\left(-\mu+a_{7}\left(|c|\left|z\left(\theta_{l} \omega\right)\right|+|c|^{2}\left|z\left(\theta_{l} \omega\right)\right|^{2}\right) d l\right.} \\
& +\frac{1}{I} \frac{3}{\mu} c_{9}\left(M_{0}^{2}\left(\theta_{-t} \omega\right)+M_{0}^{p+2}\left(\theta_{-t} \omega\right)+1\right) e^{-\frac{2 \mu}{3} t+a_{7} \int_{-t}^{0}\left(|c|\left|z\left(\theta_{l} \omega\right)\right|+|c|^{2}\left|z\left(\theta_{l} \omega\right)\right|^{2}\right) d l} \\
& +\frac{1}{2 I} M_{0}^{2}(\omega) \int_{-\infty}^{0} \xi_{1}\left(\theta_{s} \omega\right) e^{\int_{s}^{0}\left(-\mu+a_{7}\left(|c|\left|z\left(\theta_{l} \omega\right)\right|+|c|^{2}\left|z\left(\theta_{l} \omega\right)\right|^{2}\right) d l\right.} d s \\
& +\gamma_{I} \int_{-\infty}^{0} e^{\int_{s}^{0}\left[-\mu+a_{7}\left(|c|\left|z\left(\theta_{l} \omega\right)\right|+|c|^{2}\left|z\left(\theta_{l} \omega\right)\right|^{2}\right)\right] d l} d s \\
& \leq c_{9}\left(1+\frac{3}{\mu}\right)\left(M_{0}^{2}\left(\theta_{-t} \omega\right)+M_{0}^{p+2}\left(\theta_{-t} \omega\right)+1\right) e^{-\frac{2 \mu}{3} t+a_{7} \int_{-t}^{0}\left(|c|\left|z\left(\theta_{l} \omega\right)\right|+|c|^{2}\left|z\left(\theta_{l} \omega\right)\right|^{2}\right)} d l \\
& +\left(\frac{c_{6}}{I}+\gamma_{I}\right) K_{2}(\omega)
\end{aligned}
$$

By (3), we have

$$
\lim _{t \rightarrow+\infty}\left(\frac{2 \mu}{3}-\frac{a_{7}}{t} \int_{-t}^{0}\left(|c|\left|z\left(\theta_{l} \omega\right)\right|+|c|^{2}\left|z\left(\theta_{l} \omega\right)\right|^{2}\right) d s\right) \geq \frac{\mu}{6}>0
$$

Since $M_{0}^{2}(\omega)+M_{0}^{p+2}(\omega)+1$ is tempered, we have

$$
\left(M_{0}^{2}\left(\theta_{-t} \omega\right)+M_{0}^{p+2}\left(\theta_{-t} \omega\right)+1\right) e^{\left.-\frac{2 \mu}{3} t+a_{7} \int_{-t}^{0}\left(|c|\left|z\left(\theta_{l} \omega\right)\right|+|c|^{2}\left|z\left(\theta_{l} \omega\right)\right|^{2}\right)\right) d l} \rightarrow 0
$$

as $t \rightarrow+\infty$. Hence, by (41)-(42), for any $v>0, I \in \mathbb{N}$, there exists $T_{v}(\omega)>0$, such that

$$
\sum_{|i|>2 I}\left|\varphi_{i}\left(\tau, \tau-t, \theta_{-\tau} \omega, \varphi_{\tau-t}\right)\right|_{H}^{2} \leq v+\left(\frac{c_{6}}{I}+\gamma_{I}\right) K_{2}(\omega), \quad t \geq T_{v}(\omega), I \in \mathbb{N}
$$

This completes the proof. 
It follows directly from Lemmas 2.1 and 2.2 and Theorem 3.6 in [1], Theorem 3.3 in [10] that

Theorem 2.2 The cocycle $\Phi$ possesses a random attractor $R=\{R(\tau, \omega)\}_{\tau \in \mathbb{R}, \omega \in \Omega}$ in $D(H)$ with the properties: for every $\tau \in R, \omega \in \Omega$, (i) $R(\tau, \omega) \subseteq D_{0}(\omega)$; (ii) $R(\tau, \omega)$ is compact in $H$ and measurable in $\omega$; (iii) $\Phi(t, \tau, \omega, R(\tau, \omega))=R\left(t+\tau, \theta_{t} \omega\right), \forall t \geq 0$; (iv) for every $B=$ $\{B(\tau, \omega)\}_{\tau \in \mathbb{R}, \omega \in \Omega} \in D(H), \lim _{t \rightarrow+\infty} d_{h}\left(\Phi\left(t, \tau-t, \theta_{-t} \omega\right) B\left(\tau-t, \theta_{-t} \omega\right), R(\tau, \omega)\right)=0$.

\subsection{Existence of random exponential attractor}

In this subsection, we prove the existence of a random exponential attractor for $\Phi$ based on Theorem 2.1. Obviously, the family of tempered closed random subsets $D_{0}=$ $\left.\left\{D_{0}(\omega)\right\}\right\}_{\tau \in \mathbb{R}, \omega \in \Omega}$ satisfies condition (B1) in Theorem 2.1.

Taking a small enough positive constant $v_{0}>0$ such that

$$
\frac{32 a_{1}^{2} v_{0}}{\alpha \underline{\lambda}^{2} \bar{\rho}}+\frac{a_{1}^{2} 2^{p+4} v_{0}^{p}}{\alpha \underline{\lambda}^{p+1} \bar{\rho}^{p}} \leq \frac{\varepsilon}{2}
$$

For any $\omega \in \Omega$, set

$$
\begin{aligned}
T_{\nu_{0}}(\omega) & =\min \left\{t: c_{9}\left(1+\frac{3}{\mu}\right) M\left(\theta_{-t} \omega\right) e^{\left.-\frac{2 \mu}{3} t+a_{7} \int_{-t}^{0}\left(|c|\left|z\left(\theta_{l} \omega\right)\right|+|c|^{2}\left|z\left(\theta_{l} \omega\right)\right|^{2}\right)\right) d l} \leq v_{0}\right\} \\
& <+\infty
\end{aligned}
$$

where $M(\omega)=M_{0}^{2}(\omega)+M_{0}^{p+2}(\omega)+1$.

For any $\tau \in \mathbb{R}, \omega \in \Omega$, define a tempered bounded random subset $\mathcal{X}_{1}(\tau, \omega)$ of $H$ as

$$
\mathcal{X}_{1}(\tau, \omega)=\bigcup_{s \geq \max \left\{T_{D_{0}}(\omega), T_{\nu_{0}}(\omega)\right\}} \varphi\left(\tau, \tau-s, \theta_{-\tau} \omega, D_{0}\left(\theta_{-s} \omega\right)\right) \subseteq D_{0}(\omega) \subset H .
$$

Now let us show that $\left\{\mathcal{X}_{1}(\tau, \omega)\right\}_{\tau \in \mathbb{R}, \omega \in \Omega}$ satisfies (B2) of Theorem 2.1.

For any $\tau \in \mathbb{R}, \omega \in \Omega, t \geq 0$, and $\varphi_{j, \tau-t}\left(\theta_{-\tau} \omega\right) \in \mathcal{X}_{1}\left(\tau-t, \theta_{-t} \omega\right) \subseteq D_{0}\left(\theta_{-t} \omega\right), j=1$, 2. Let $\varphi_{j}(r)=\varphi\left(r, \tau-t, \theta_{-\tau} \omega, \varphi_{j, \tau-t}\left(\theta_{-\tau} \omega\right)\right)=\left(u_{j}(r), v_{j}(r)\right), j=1,2, \psi(r)=\varphi_{1}(r)-\varphi_{2}(r)=(\xi(r), \zeta(r))$, $r \geq \tau-t$, then

$$
\left\{\begin{array}{l}
\dot{\psi}+L \psi=F\left(\varphi_{1}, \theta_{r-\tau} \omega\right)-F\left(\varphi_{2}, \theta_{r-\tau} \omega\right), \\
\psi_{\tau}(\omega)=\left(\xi_{\tau}, \zeta_{\tau}\right)=\left(u_{1 \tau}-u_{2 \tau}, \nu_{1 \tau}-v_{2 \tau}\right), \quad r \geq \tau-t,
\end{array}\right.
$$

where

$$
\begin{aligned}
F\left(\varphi_{1}, \theta_{r-\tau} \omega\right)-F\left(\varphi_{2}, \theta_{r-\tau} \omega\right) & \\
\quad= & \left.\begin{array}{c}
c z\left(\theta_{r-\tau} \omega\right) \xi \\
(2 \varepsilon-c z) c z \xi-\gamma c A z \xi-c z \zeta-f\left(u_{1}, t\right)+f\left(u_{2}, t\right)
\end{array}\right) .
\end{aligned}
$$

By Lemma 2.2 and (44), we have

$$
\left\|\varphi_{1}(r)\right\|_{H} \leq M_{0}\left(\theta_{r-\tau} \omega\right), \quad\left\|\varphi_{2}(r)\right\|_{H} \leq M_{0}\left(\theta_{r-\tau} \omega\right), \quad \forall r \geq \tau-t
$$


and

$$
\sum_{|i| \geq 2 I}\left|\varphi_{i}\left(r, \tau-t, \theta_{-\tau} \omega, \varphi_{\tau-t}\left(\theta_{-\tau} \omega\right)\right)\right|_{H}^{2} \leq v_{0}+\left(\frac{c_{6}}{I}+\gamma_{I}\right) K_{2}\left(\theta_{r-\tau} \omega\right), \quad I \in \mathbb{N}, r \geq \tau-t
$$

Lemma 2.3 For every $\tau \in \mathbb{R}, t \geq 0, \omega \in \Omega$ and $\varphi_{j, \tau-t}\left(\theta_{-\tau} \omega\right) \in \mathcal{X}_{1}\left(\tau-t, \theta_{-t} \omega\right), j=1$, 2, there exist random variables $C_{2}(\omega)>0, C_{3}(\omega)>0$, such that

$$
\begin{aligned}
& \left\|\varphi\left(\tau, \tau-t, \theta_{-\tau} \omega\right) \varphi_{1, \tau-t}\left(\theta_{-\tau} \omega\right)-\varphi\left(\tau, \tau-t, \theta_{-\tau} \omega\right) \varphi_{2, \tau-t}\left(\theta_{-\tau} \omega\right)\right\|_{H} \\
& \quad \leq e^{\int_{-t}^{0} C_{2}\left(\theta_{s} \omega\right) d s}\left\|\varphi_{1, \tau-t}\left(\theta_{-\tau} \omega\right)-\varphi_{2, \tau-t}\left(\theta_{-\tau} \omega\right)\right\|_{H}
\end{aligned}
$$

and

$$
\begin{aligned}
& \sum_{|i|>4 I}\left|\left(\varphi\left(r, \tau-t, \theta_{-\tau} \omega, \varphi_{1, \tau-t}\left(\theta_{-\tau} \omega\right)\right)-\varphi\left(r, \tau-t, \theta_{-\tau} \omega, \varphi_{2, \tau-t}\left(\theta_{-\tau} \omega\right)\right)\right)_{i}\right|_{H}^{2} \\
& \leq\left(e^{-\frac{\varepsilon}{4} t+\int_{-t}^{0} C_{3}\left(\theta_{s} \omega\right) d s}+\frac{\hat{\delta}_{I}}{2} e^{\int_{-t}^{0} C_{2}\left(\theta_{s} \omega\right) d s}\right)^{2}\|\psi(\tau-t)\|_{H}^{2}, \quad \forall I \in \mathbb{N}
\end{aligned}
$$

where

$$
\hat{\delta}_{I}=\frac{2}{\sqrt[4]{\varepsilon}} \sqrt{\frac{1}{I}+\gamma_{I}+\frac{1}{I^{p}}+\gamma_{I}^{p}}
$$

Proof Taking the inner product $(\cdot, \cdot)_{H}$ of $(45)$ with $\psi(r)$, we find that

$$
\begin{aligned}
& \frac{d\|\psi(r)\|_{H}^{2}}{d t} \\
& \leq\left(-\varepsilon+2\left(1+\frac{\varepsilon}{\sqrt{\underline{\lambda}}}\right)|c|\left|z\left(\theta_{r-\tau} \omega\right)\right|+\left(\frac{8 a_{5}^{2}}{\underline{\lambda} \alpha}+\frac{1}{\sqrt{\underline{\lambda}}}\right)|c|^{2}\left|z\left(\theta_{r-\tau} \omega\right)\right|^{2}\right)\|\psi(r)\|_{H}^{2} \\
& \quad+\frac{2}{\alpha}\left\|f\left(u_{2}, r\right)-f\left(u_{1}, r\right)\right\|_{\rho}^{2} .
\end{aligned}
$$

By (A2) and (46), we have

$$
\left\|f\left(u_{2}, r\right)-f\left(u_{1}, r\right)\right\|_{\rho}^{2}=\sum_{i \in \mathbb{Z}} \rho_{i}\left|f_{i}\left(u_{2, i}, r\right)-f_{i}\left(u_{1, i}, r\right)\right|^{2} \leq \frac{C_{0}\left(\theta_{r-\tau} \omega\right)}{\underline{\lambda}}\|\psi(r)\|_{H}^{2},
$$

where

$$
c_{10}=a_{1}^{2}\left(\frac{16}{\underline{\lambda}}+\frac{2^{2(p-1)}}{\underline{\lambda}^{p-1}}\right), \quad C_{0}(\omega)=c_{10} M_{0}^{2}(\omega)\left(1+M_{0}^{2(p-1)}(\omega)\right) .
$$

Thus,

$$
\frac{d\|\psi(r)\|_{H}^{2}}{d t} \leq\left(-\varepsilon+2 C_{1}\left(\theta_{r-\tau} \omega\right)\right)\|\psi(r)\|_{H}^{2},
$$

where

$$
C_{1}(\omega)=\left(1+\frac{\varepsilon}{\sqrt{\underline{\lambda}}}\right)|c||z(\omega)|+\left(\frac{4 a_{5}^{2}}{\underline{\lambda} \alpha}+\frac{1}{2 \sqrt{\underline{\lambda}}}\right)|c|^{2}|z(\omega)|^{2}+\frac{C_{0}(\omega)}{\alpha \underline{\lambda}} .
$$


By applying the Gronwall inequality to (52) on $[\tau-t, \tau]$, we have

$$
\|\psi(\tau)\|_{H}^{2} \leq e^{\int_{-t}^{0}\left(-\varepsilon+2 C_{1}\left(\theta_{s} \omega\right)\right) d s}\|\psi(\tau-t)\|_{H}^{2} \leq e^{2 \int_{-t}^{0} C_{1}\left(\theta_{s} \omega\right) d s}\|\psi(\tau-t)\|_{H}^{2} .
$$

Let $I \in \mathbb{N}$ and $M$ be a suitable large integer, and set

$$
\omega=\left(\omega_{i}\right)_{i \in \mathbb{Z}}=\left(\eta\left(\frac{|i|}{M}\right) \xi_{i}, \eta\left(\frac{|i|}{M}\right) \zeta_{i}\right)=\left(p_{i}, q_{i}\right) .
$$

Taking the inner product $(\cdot, \cdot)_{H}$ of (45) with $\omega$, we find that, for $r \geq \tau-t$,

$$
(\dot{\psi}, \omega)_{H}+(L \psi, \omega)_{H}=\left(F\left(\varphi_{1}(r), \theta_{r-\tau} \omega\right)-F\left(\varphi_{2}(r), \theta_{r-\tau} \omega\right), \omega\right)_{H}
$$

Similar to (30)-(35), and by (A2), we obtain, for $r \geq \tau-t$,

$$
\begin{aligned}
& 2\left(f\left(u_{2}, r\right)-f\left(u_{1}, r\right), q\right)_{\rho} \\
& \leq \frac{\alpha}{4} \sum_{i \in \mathbb{Z}} \eta\left(\frac{|i|}{M}\right) \rho_{i}\left|\zeta_{i}\right|^{2}+\frac{16 a_{1}^{2}}{\alpha} \sum_{i \in \mathbb{Z}} \eta\left(\frac{|i|}{M}\right) \rho_{i}\left(\left|u_{1, i}\right|^{2}+\left|u_{2, i}\right|^{2}\right)\left|\xi_{i}\right|^{2} \\
& \quad+\frac{16 a_{1}^{2}}{\alpha} \sum_{i \in \mathbb{Z}} \eta\left(\frac{|i|}{M}\right) \rho_{i}\left(\left|u_{1, i}\right|^{2 p}+\left|u_{2, i}\right|^{2 p}\right)\left|\xi_{i}\right|^{2} .
\end{aligned}
$$

By (47),

$$
\begin{aligned}
& \sum_{|i| \geq 2 I}\left[\left|u_{1, i}\right|^{2}+\left|u_{2, i}\right|^{2}\right] \leq \frac{2}{\underline{\lambda} \bar{\rho}} v_{0}+\frac{2}{\underline{\lambda} \bar{\rho}}\left(\frac{c_{6}}{I}+\gamma_{I}\right) K_{2}\left(\theta_{r-\tau} \omega\right), \\
& \sum_{|i| \geq 2 I}\left[\left|u_{1, i}\right|^{2 p}+\left|u_{2, i}\right|^{2 p}\right] \leq \frac{2^{p}}{\underline{\lambda}^{p} \bar{\rho}^{p}} v_{0}^{p}+\frac{2^{p}}{\underline{\lambda}^{p} \bar{\rho}^{p}}\left(\frac{c_{6}}{I}+\gamma_{I}\right)^{p} K_{2}^{p}\left(\theta_{r-\tau} \omega\right) .
\end{aligned}
$$

Then we have, for $M \geq 2 I$,

$$
\begin{aligned}
& 2\left(f\left(u_{2}, r\right)-f\left(u_{1}, r\right), q\right)_{\rho} \\
& \leq \frac{\alpha}{4} \sum_{i \in \mathbb{Z}} \eta\left(\frac{|i|}{M}\right) \rho_{i}\left|\zeta_{i}\right|^{2}+\left(\frac{32 a_{1}^{2} v_{0}}{\alpha \underline{\lambda}^{2} \bar{\rho}}+\frac{a_{1}^{2} 2^{p+4} v_{0}^{p}}{\alpha \underline{\lambda}^{p+1} \bar{\rho}^{p}}\right) \sum_{i \in \mathbb{Z}} \eta\left(\frac{|i|}{M}\right)\left|\psi_{i}\right|_{H}^{2} \\
&+\frac{32 a_{1}^{2}}{\alpha \underline{\lambda} \bar{\rho}}\left(\frac{c_{6}}{I}+\gamma_{I}\right) K_{2}\left(\theta_{r-\tau} \omega\right) \sum_{i \in \mathbb{Z}} \eta\left(\frac{|i|}{M}\right) \rho_{i}\left|\xi_{i}\right|^{2} \\
&+\frac{a_{1}^{2} 2^{p+4}}{\alpha \underline{\lambda}^{p} \bar{\rho}^{p}}\left(\frac{c_{6}}{I}+\gamma_{I}\right)^{p} K_{2}^{p}\left(\theta_{r-\tau} \omega\right) \sum_{i \in \mathbb{Z}} \eta\left(\frac{|i|}{M}\right) \rho_{i}\left|\xi_{i}\right|^{2},
\end{aligned}
$$

where $\left|\psi_{i}\right|_{H}^{2}=\rho_{i}\left[\delta(D \xi)_{i}^{2}+\lambda_{i} \xi_{i}^{2}+\zeta_{i}^{2}\right]$. Thus, by (43) and the above inequality, we have, for $M \geq 2 I$,

$$
\begin{aligned}
& \frac{d}{d t} \sum_{i \in \mathbb{Z}} \eta\left(\frac{|i|}{M}\right)\left|\psi_{i}(r)\right|_{H}^{2}+\frac{\varepsilon}{2} \sum_{i \in \mathbb{Z}} \eta\left(\frac{|i|}{M}\right)\left|\psi_{i}(r)\right|_{H}^{2} \\
& \quad \leq\left(\left(2+\frac{2 \varepsilon}{\sqrt{\underline{\lambda}}}\right)|c|\left|z\left(\theta_{r-\tau} \omega\right)\right|+\left(\frac{8 a_{5}^{2}}{\underline{\lambda} \alpha}+\frac{1}{\sqrt{\underline{\lambda}}}\right)|c|^{2}\left|z\left(\theta_{r-\tau} \omega\right)\right|^{2}\right) \sum_{i \in \mathbb{Z}} \eta\left(\frac{|i|}{M}\right)\left|\psi_{i}(r)\right|_{H}^{2}
\end{aligned}
$$




$$
\begin{aligned}
& +c_{11}\left(\frac{1}{I}+\gamma_{I}+\frac{1}{I^{p}}+\gamma_{I}^{p}\right) \cdot\left[\xi_{1}\left(\theta_{r-\tau} \omega\right)+K_{2}^{p}\left(\theta_{r-\tau} \omega\right)\right] \\
& \times e^{2 \int_{r}^{\tau-t} C_{2}\left(\theta_{s-\tau} \omega\right) d s}\|\psi(\tau-t)\|_{H^{\prime}}^{2}
\end{aligned}
$$

where

$$
c_{11}=\max \left\{\frac{1}{2}, \frac{32 a_{1}^{2}}{\alpha \underline{\lambda}^{2} \bar{\rho}}, \frac{32 c_{6} a_{1}^{2}}{\alpha \underline{\lambda}^{2} \bar{\rho}}, \frac{2^{2 p+3} c_{6} a_{1}^{2}}{\alpha \underline{\lambda}^{p+1} \bar{\rho}^{p}}, \frac{2^{2 p+3} a_{1}^{2}}{\alpha \underline{\lambda}^{p+1} \bar{\rho}^{p}}\right\} .
$$

By applying the Gronwall inequality to (57) on $[\tau-t, \tau](t \geq 0)$, we obtain

$$
\begin{aligned}
\sum_{i \in \mathbb{Z}} & \eta\left(\frac{|i|}{M}\right)\left|\psi_{i}(\tau)\right|_{H}^{2} \\
\leq & \|\psi(\tau-t)\|_{E}^{2} e^{\int_{-t}^{0}\left[-\frac{\varepsilon}{2}+2\left(1+\frac{\varepsilon}{\sqrt{\underline{\lambda}}}\right)|c|\left|z\left(\theta_{s} \omega\right)\right|+\left(\frac{8 a_{5}^{2}}{\underline{\lambda} \alpha}+\frac{1}{\sqrt{\underline{\lambda}}}\right)|c|^{2}\left|z\left(\theta_{s} \omega\right)\right|^{2}\right] d s} \\
& +\left(\frac{1}{I}+\gamma_{I}+\frac{1}{I^{p}}+\gamma_{I}^{p}\right)\|\psi(\tau-t)\|_{H}^{2} \\
& \times e^{\int_{-t}^{0} 2\left[C_{1}\left(\theta_{s} \omega\right)+\left(1+\frac{\varepsilon}{\sqrt{\lambda}}\right)|c|\left|z\left(\theta_{s} \omega\right)\right|+\left(\frac{4 a_{5}^{2}}{\underline{\lambda} \alpha}+\frac{1}{2 \sqrt{\underline{\lambda}}}\right)|c|^{2}\left|z\left(\theta_{s} \omega\right)\right|^{2}\right] d s} \\
& \times \int_{-t}^{0} c_{11}\left(\xi_{1}\left(\theta_{r} \omega\right)+K_{2}^{p}\left(\theta_{r} \omega\right)\right) e^{\frac{\varepsilon}{2} r} d r .
\end{aligned}
$$

Since $\sqrt{x} \leq e^{x}$ for all $x \geq 0$, it follows that

$$
\begin{aligned}
& \int_{-t}^{0} c_{11}\left(\xi_{1}\left(\theta_{r} \omega\right)+K_{2}^{p}\left(\theta_{r} \omega\right)\right) e^{\frac{\varepsilon}{2} r} d r \\
& \leq\left[\int_{-t}^{0} c_{11}^{2}\left(\xi_{1}\left(\theta_{r} \omega\right)+K_{2}^{p}\left(\theta_{r} \omega\right)\right)^{2} d r\right]^{\frac{1}{2}}\left[\int_{-t}^{0} e^{\varepsilon r} d r\right]^{\frac{1}{2}} \\
& \quad \leq \frac{1}{\sqrt{\varepsilon}} e^{\int_{-t}^{0} c_{11}^{2}\left(\xi_{1}\left(\theta_{r} \omega\right)+K_{2}^{p}\left(\theta_{r} \omega\right)\right)^{2} d r}
\end{aligned}
$$

By $M \geq 2 I$ and (58),

$$
\begin{aligned}
& \sum_{|i|>4 I}\left|\psi_{i}(\tau)\right|_{H}^{2} \\
& \quad \leq \sum_{|i| \in \mathbb{Z}} \eta\left(\frac{|i|}{M}\right)\left|\psi_{i}(\tau)\right|_{H}^{2} \\
& \quad \leq\left(e^{\int_{-t}^{0} 2\left(-\frac{\varepsilon}{4}+C_{3}\left(\theta_{s} \omega\right)\right) d s}+\hat{\delta}_{I}^{2} e^{2 \int_{-t}^{0} C_{2}\left(\theta_{s} \omega\right) d s}\right)\|\psi(\tau-t)\|_{H}^{2},
\end{aligned}
$$

where

$$
\begin{aligned}
& C_{2}(\omega)=C_{1}(\omega)+C_{3}(\omega)+c_{11}^{2}\left(\xi_{1}^{2}(\omega)+K_{2}^{2 p}(\omega)\right), \\
& C_{3}(\omega)=\left(1+\frac{\varepsilon}{\sqrt{\underline{\lambda}}}\right)|c||z(\omega)|+\left(\frac{4 a_{5}^{2}}{\underline{\lambda} \alpha}+\frac{1}{2 \sqrt{\underline{\lambda}}}\right)|c|^{2}|z(\omega)|^{2},
\end{aligned}
$$

thus, (49) holds. By (54) and $C_{2}(\omega) \geq C_{1}(\omega)$, it follows that (48) holds. The proof is completed. 
Lemma 2.4 Let the coefficient $c$ of the random term in (2) satisfy

$$
|c|^{2}<\min \left\{\frac{\mu^{2} \pi \alpha}{64^{2} a_{7}^{2}}, \frac{\alpha \mu}{32 a_{7}}, \frac{\alpha^{2}}{64 p a_{7}}, \frac{\alpha^{3}}{32^{2} p^{2} a_{7}^{2}}\right\}, \quad \mu=\min \left\{\varepsilon, \frac{\varepsilon}{2 a_{2}}, 1\right\},
$$

then

$$
0 \leq \mathbf{E}\left[C_{3}(\omega)\right] \leq \frac{\varepsilon}{64}, \quad 0 \leq \mathbf{E}\left[C_{2}(\omega)\right], \mathbf{E}\left[C_{2}^{2}(\omega)\right]<\infty
$$

Proof From $[13,16]$, the Ornstein-Uhlenbeck stationary process $z\left(\theta_{t} \omega\right)$ satisfies

$$
\begin{aligned}
& \mathbf{E}\left[\left|z\left(\theta_{s} \omega\right)\right|^{r}\right]=\frac{\Gamma\left(\frac{1+r}{2}\right)}{\sqrt{\pi \alpha^{r}}} \quad(\forall r \geq 0), \\
& \left\{\begin{array}{l}
\mathbf{E}\left[e^{\int_{\tau}^{\tau+t}\left|z\left(\theta_{s} \omega\right)\right|^{2} d s}\right] \leq e^{\frac{\zeta}{\alpha} t}, \quad 0 \leq 2 \zeta \leq \alpha^{2}, \tau \in \mathbb{R}, t \geq 0, \\
\mathbf{E}\left[e^{\int_{\tau}^{\tau+t}\left|z\left(\theta_{s} \omega\right)\right| d s}\right] \leq e^{\frac{\zeta}{\sqrt{\alpha}} t}, \quad 0 \leq \zeta^{2} \leq \alpha^{3}, \tau \in \mathbb{R}, t \geq 0,
\end{array}\right.
\end{aligned}
$$

where $\Gamma(\cdot)$ is the Gamma function. It is easy to see that (61) implies (8). By (3), (59) and (60), we obtain

$$
\mathbf{E}\left[C_{3}(\omega)\right] \leq \frac{a_{7}|c|}{2 \sqrt{\pi \alpha}}+\frac{a_{7}|c|^{2}}{4 \alpha} \leq \frac{1}{16} \frac{\varepsilon}{4} .
$$

By (7), (61) and (63)-(64),

$$
\begin{aligned}
\mathbf{E}\left[M_{0}^{4 p}(\omega)\right] & \leq c_{5}^{2 p}\left(\int_{-\infty}^{0} e^{\frac{p \mu}{2 p-1} s} d s\right)^{2 p-1} \int_{-\infty}^{0} e^{p \mu s} \mathbf{E} e^{2 p a_{7} \int_{s}^{0}\left(|c|\left|z\left(\theta_{l} \omega\right)\right|+|c|^{2}|z|^{2}\left(\theta_{l} \omega\right)\right) d l} d s \\
& \leq c_{5}^{2 p}\left(\frac{2 p-1}{p \mu}\right)^{2 p-1} \int_{-\infty}^{0} e^{p \mu s}\left(\mathbf{E} e^{4 p a_{7}|c| \int_{s}^{0}\left|z\left(\theta_{l} \omega\right)\right| d l}+\mathbf{E} e^{4 p a_{7}|c|^{2} \int_{s}^{0}|z|^{2}\left(\theta_{l} \omega\right) d l}\right) d s \\
& \leq \frac{c_{5}^{2 p}}{p}\left(\frac{2 p-1}{p \mu}\right)^{2 p-1}\left(\frac{1}{\mu-\frac{4|c| a_{7}}{\sqrt{\alpha}}}+\frac{1}{\mu-\frac{4|c|^{2} a_{7}}{\alpha}}\right)=k_{1} .
\end{aligned}
$$

Then, by (51), (53) and (66),

$$
\begin{aligned}
\mathbf{E}\left[C_{1}^{2}(\omega)\right] \leq & 3 \mathbf{E}\left(\left(1+\frac{\varepsilon}{\sqrt{\lambda}}\right)^{2}|c|^{2}|z(\omega)|^{2}+\left(\frac{4 a_{5}^{2}}{\underline{\lambda} \alpha}+\frac{1}{2 \sqrt{\lambda}}\right)^{4}|c|^{4}|z(\omega)|^{4}\right) \\
& +3 \mathbf{E}\left(\frac{2 c_{10}^{2}}{\alpha^{2} \underline{\lambda}^{2}}\left(M_{0}^{4}(\omega)+M_{0}^{4 p}(\omega)\right)\right) \\
\leq & 3\left(\left(1+\frac{\varepsilon}{\sqrt{\underline{\lambda}}}\right)^{2} \frac{|c|^{2}}{2 \alpha}+\left(\frac{4 a_{6}^{2}}{\underline{\lambda} \alpha}+\frac{1}{2 \sqrt{\underline{\lambda}}}\right)^{4} \frac{\Gamma\left(\frac{5}{2}\right)|c|^{4}}{\alpha^{2} \sqrt{\pi}}\right) \\
& +3\left(\frac{2 c_{10}^{2}}{\alpha^{2} \underline{\lambda}^{2}}\left(M_{0}^{4}(\omega)+M_{0}^{4 p}(\omega)\right)\right)=k_{2} .
\end{aligned}
$$

Similar to (66), we have

$$
\mathbf{E}\left[K_{0}^{4 p}(\omega)\right]=\mathbf{E}\left(\int_{-\infty}^{0} e^{\mu s+a_{7} \int_{s}^{0}\left[|c|\left|z\left(\theta_{l} \omega\right)\right|+|c|^{2}|z|^{2}\left(\theta_{l} \omega\right)\right] d l} d s\right)^{4 p}
$$




$$
\begin{aligned}
& \leq\left(\frac{4 p-1}{2 p \mu}\right)^{4 p-1} \int_{-\infty}^{0} e^{2 p \mu s}\left(\mathbf{E} e^{8 a_{7} p|c| \int_{s}^{0}\left|z\left(\theta_{l} \omega\right)\right| d l}+\mathbf{E} e^{8 a_{7} p c^{2} \int_{s}^{0}|z|^{2}\left(\theta_{l} \omega\right) d l}\right) d s \\
& \leq \frac{1}{2 p}\left(\frac{4 p-1}{2 p \mu}\right)^{4 p-1}\left(\frac{1}{\mu-\frac{4|c| a_{7}}{\sqrt{\alpha}}}+\frac{1}{\mu-\frac{4|c|^{2} a_{7}}{\alpha}}\right)=k_{3} .
\end{aligned}
$$

From (28),

$$
\begin{aligned}
& \mathbf{E}\left[\xi_{1}^{4}(\omega)\right] \leq \mathbf{E}\left[c_{12}\left(1+|z(\omega)|^{4}\right)\right] \leq c_{12}\left(1+\frac{\Gamma\left(\frac{5}{2}\right)}{\alpha^{2} \sqrt{\pi}}\right)=k_{4}, \\
& \mathbf{E}\left[\xi_{1}^{16 p}(\omega)\right] \leq \mathbf{E}\left[c_{13}\left(1+|z(\omega)|^{16 p}\right)\right] \leq c_{13}\left(1+\frac{\Gamma\left(\frac{16 p+1}{2}\right)}{\alpha^{8 p} \sqrt{\pi}}\right)=k_{5} .
\end{aligned}
$$

Thus, by (28) and (70),

$$
\begin{aligned}
\mathbf{E}\left[K_{1}^{8 p}(\omega)\right]= & \mathbf{E}\left(\int_{-\infty}^{0} \xi_{1}\left(\theta_{s} \omega\right) e^{\mu s+a_{7} \int_{s}^{0}\left(|c|\left|z\left(\theta_{l} \omega\right)\right|+|c|^{2}\left|z\left(\theta_{l} \omega\right)\right|^{2}\right) d l} d s\right)^{8 p} \\
\leq & \left(\int_{-\infty}^{0} e^{\frac{4 p}{8 p-1} \mu s} d s\right)^{8 p-1} \mathbf{E} \int_{-\infty}^{0} \xi_{1}^{8 p}\left(\theta_{s} \omega\right) e^{4 p \mu s+8 p a_{7} \int_{s}^{0}\left(|c|\left|z\left(\theta_{l} \omega\right)\right|+|c|^{2}\left|z\left(\theta \theta_{l} \omega\right)\right|^{2}\right) d l} d s \\
\leq & \left(\frac{8 p-1}{4 p \mu}\right)^{8 p-1} \times \int_{-\infty}^{0} e^{4 p \mu s}\left(\mathbf{E} e^{32 p a a_{7}|c| \int_{s}^{0}\left|z\left(\theta_{l} \omega\right)\right| d l}+\mathbf{E} e^{32 p a a_{7}|c|^{2} \int_{s}^{0}\left|z\left(\theta_{l} \omega\right)\right|^{2} d l}\right) d s \\
& +\left(\frac{8 p-1}{4 p \mu}\right)^{8 p-1} \int_{-\infty}^{0} e^{4 p \mu s} \mathbf{E} \xi_{1}^{16 p}\left(\theta_{s} \omega\right) d s \\
\leq & \frac{1}{4 p}\left(\frac{8 p-1}{4 p \mu}\right)^{8 p-1}\left(\frac{1}{\mu-\frac{8 a_{7}|c|}{\sqrt{\alpha}}}+\frac{1}{\mu-\frac{8 a_{7}|c|^{2}}{\alpha}}+\frac{k_{5}}{\mu}\right)=k_{6}, \\
\mathbf{E}\left[K_{2}^{4 p}(\omega)\right] \leq & 2^{4 p-1}\left(\mathbf{E}\left[K_{0}^{4 p}(\omega)\right]+\mathbf{E}\left[K_{0}^{8 p}(\omega)\right]+\mathbf{E}\left[K_{1}^{8 p}(\omega)\right]\right)=k_{7} .
\end{aligned}
$$

By (59), (67), (69) and (72), we have

$$
0 \leq \mathbf{E}\left[C_{2}^{2}(\omega)\right] \leq c_{14}\left(1+k_{2}+k_{4}+k_{7}\right)<\infty
$$

Since $0 \leq \mathbf{E}\left[C_{2}(\omega)\right] \leq \frac{1}{2}\left[1+\mathbf{E}\left(C_{2}^{2}(\omega)\right)\right]$, it follows that $0 \leq \mathbf{E}\left[C_{2}(\omega)\right]<\infty$. The proof is completed.

Lemma 2.5 For any $\tau \in \mathbb{R}, \omega \in \Omega$,

$$
\left\{\begin{array}{l}
\lim _{t \rightarrow 0^{+}} \sup _{\varphi \in \mathcal{X}_{1}(\tau, \omega)}\|\Phi(t, \tau, \omega) \varphi-\varphi\|_{H}=0, \\
\lim _{t \rightarrow 0^{+}} \sup _{\varphi \in \mathcal{X}_{1}\left(\tau-t, \theta_{-} \omega\right)}\left\|\Phi\left(0, \tau-t, \theta_{-t} \omega\right) \varphi-\varphi\right\|_{H}=0 .
\end{array}\right.
$$

Proof By (5), (6) and (44), it follows that, for $\varphi \in \mathcal{X}_{1}(\tau, \omega)$ and $t \geq 0$,

$$
\begin{aligned}
& \left\|F\left(\varphi(r), \theta_{r-\tau} \omega\right)\right\|_{H}^{2} \\
& \quad \leq|c|^{2}\left|z\left(\theta_{r-\tau} \omega\right)\right|^{2} M_{0}^{2}\left(\theta_{r-\tau} \omega\right)+\frac{5\left(2 c \varepsilon z\left(\theta_{r-\tau} \omega\right)-|c|^{2}\left|z\left(\theta_{r-\tau} \omega\right)\right|^{2}\right)^{2}}{\sqrt{\lambda}} M_{0}^{2}\left(\theta_{r-\tau} \omega\right) \\
& \quad+5|c|^{2}\left|z\left(\theta_{r-\tau} \omega\right)\right|^{2} M_{0}^{2}\left(\theta_{r-\tau} \omega\right)+5|c|^{2} a_{5}^{2}\left|z\left(\theta_{r-\tau} \omega\right)\right|^{2} M_{0}^{2}\left(\theta_{r-\tau} \omega\right)
\end{aligned}
$$




$$
+5 a_{1}^{2}\left(1+M_{0}^{p-1}\left(\theta_{r-\tau} \omega\right)\right)^{2} M_{0}^{4}\left(\theta_{r-\tau} \omega\right)+5\|g\|_{\rho}^{2}
$$

and

$$
\begin{aligned}
\|L \varphi(r)\|_{H}^{2} \leq & \|\varepsilon u-v\|_{\delta, \lambda, \rho}^{2}+5 \lambda^{2}\|u\|_{\rho}^{2}+5|\varepsilon|(\alpha-\varepsilon) \mid\|u\|_{\rho}^{2} \\
& +5 \delta\|A u\|_{\rho}^{2}+5(\alpha-\varepsilon)^{2}\|v\|_{\rho}^{2}+5 \gamma\|A v\|_{\rho}^{2} \\
\leq & c_{15} M_{0}^{2}\left(\theta_{r-\tau} \omega\right)
\end{aligned}
$$

therefore,

$$
\begin{aligned}
& \|\Phi(t, \tau, \omega) \varphi-\varphi\|_{H}^{2} \\
& \leq t \int_{\tau}^{\tau+t}\left\|F\left(\varphi(r), \theta_{r-\tau} \omega\right)-L \varphi(r)\right\|_{H}^{2} d r \\
& \leq 2 t \int_{0}^{t}\left[\frac{5\left(2 c \varepsilon z\left(\theta_{r} \omega\right)-|c|^{2}\left|z\left(\theta_{r} \omega\right)\right|^{2}\right)^{2}}{\sqrt{\lambda}}+c_{16}|c|^{2}\left|z\left(\theta_{r} \omega\right)\right|^{2}\right] M_{0}^{2}\left(\theta_{r} \omega\right) d r \\
& \quad+2 t \int_{0}^{t}\left[5 a_{1}^{2}\left(1+M_{0}^{p-1}\left(\theta_{r} \omega\right)\right)^{2} M_{0}^{4}\left(\theta_{r} \omega\right)+5\|g\|_{\rho}^{2}+c_{15} M_{0}^{2}\left(\theta_{r} \omega\right)\right] d r \\
& \rightarrow 0 \quad \text { as } t \rightarrow 0,
\end{aligned}
$$

where $c_{16}=6+5 a_{5}^{2}$, thus, $\lim _{t \rightarrow 0^{+}} \sup _{\varphi \in \mathcal{X}_{1}(\tau, \omega)}\|\Phi(t, \tau, \omega) \varphi-\varphi\|_{H}=0$.

Similarly, $\lim _{t \rightarrow 0^{+}} \sup _{\varphi \in \mathcal{X}_{1}\left(\tau-t, \theta_{-t} \omega\right)}\left\|\Phi\left(0, \tau-t, \theta_{-t} \omega\right) \varphi-\varphi\right\|_{H}=0$. The proof is completed.

From Theorem 2.1 and Lemmas 2.3-2.5, we have the following result.

Theorem 2.3 Assume that (A1)-(A4) and (43), (61) hold. Then the continuous cocycle $\{\Phi(t, \tau, \omega)\}_{t \geq 0, \tau \in \mathbb{R}, \omega \in \Omega}$ possesses a random exponential attractor $\{\mathcal{K}(\tau, \omega)\}_{\tau \in \mathbb{R}, \omega \in \Omega}$ with properties: for any $\tau \in \mathbb{R}, \omega \in \Omega$,

(i) $\mathcal{R}(\tau, \omega) \subseteq \mathcal{K}(\tau, \omega) \subseteq \overline{\mathcal{X}_{1}(\tau, \omega)}$ and $\mathcal{K}(\tau, \omega)$ is a compact set of $H$ and measurable in $\omega$.

(ii) $\Phi(t, \tau, \omega) \mathcal{K}(\tau, \omega) \subseteq \mathcal{K}\left(t+\tau, \theta_{t} \omega\right)$ for all $t \geq 0$.

(iii) There exists $I_{0}=\min \left\{I: 0<\hat{\delta}_{I} \leq \tilde{\gamma}\right\}+1 \in N$, where

$$
\tilde{\gamma}=\min \left\{\frac{1}{16}, e^{-\frac{2}{\ln \frac{3}{2}}\left(\frac{32 \ln 2}{\varepsilon}\right)^{2}\left(\mathbf{E}\left[C_{2}^{2}(\omega)\right]+\frac{\varepsilon}{4} \mathbf{E}\left[C_{2}(\omega)\right]\right)}\right\}
$$

such that

$$
\operatorname{dim}_{f} \mathcal{R}(\tau, \omega) \leq \operatorname{dim}_{f} \mathcal{K}(\tau, \omega) \leq \frac{4\left(8 I_{0}+1\right) \ln \left(\frac{2 \sqrt{16 I_{0}+2}}{\hat{\delta}_{I_{0}}}+1\right)}{\ln \frac{4}{3}}<\infty
$$

(iv) For every set $D \in \mathcal{D}(H)$, there exist a random variable $T_{\omega, D} \geq 0$ and a tempered random variable $b_{\omega, D}>0$ such that

$$
\mathrm{d}_{h}\left(\Phi(t, \tau, \omega) D(\tau, \omega), \mathcal{K}\left(t+\tau, \theta_{t} \omega\right)\right) \leq b_{\omega, D} e^{-\frac{\varepsilon \ln \frac{4}{3}}{128 \ln 2} t}, \quad t \geq T_{\omega, D}
$$


(v) For any $\tau \in \mathbb{R}, \omega \in \Omega, \lim _{t \rightarrow 0} \mathrm{~d}_{h}\left(\mathcal{K}\left(\tau+t, \theta_{t} \omega\right), \mathcal{K}(\tau, \omega)\right)=0$.

Proof Taking $t=\frac{32 \ln 2}{\varepsilon}$ in (48) and (49). From (62),

$$
0<e^{-\frac{2}{\ln \frac{3}{2}}\left(\frac{32 \ln 2}{\varepsilon}\right)^{2}\left(\mathbf{E}\left[C_{2}^{2}(\omega)\right]+\frac{\varepsilon}{4} \mathbf{E}\left[C_{2}(\omega)\right]\right)}<\infty
$$

By $\lim _{I \rightarrow+\infty} \frac{1}{I}=\lim _{I \rightarrow+\infty} \frac{1}{I^{p+1}}=0$ and $g(t), \beta(t), \beta^{\prime}(t) \in \mathbf{G}$, it follows that $\lim _{I \rightarrow+\infty} \gamma_{I}=0$ and

$$
0<\hat{\delta}_{I}=\frac{2}{\sqrt[4]{\varepsilon}} \sqrt{\frac{1}{I}+\gamma_{I}+\frac{1}{I^{p+1}}+\gamma_{I}^{p+1}} \rightarrow 0 \quad \text { as } I \rightarrow+\infty .
$$

Hence there exists a finite integer $I_{0} \in \mathbb{N}$, such that

$$
0<\hat{\delta}_{I_{0}} \leq \min \left\{\frac{1}{16}, e^{-\frac{2}{\ln \frac{3}{2}}\left(\frac{32 \ln 2}{\varepsilon}\right)^{2}\left(\mathbf{E}\left[C_{2}^{2}(\omega)\right]+\frac{\varepsilon}{4} \mathbf{E}\left[C_{2}(\omega)\right]\right)}\right\}
$$

Finally, by Theorem 2.1, the statements in Theorem 2.3 hold. The proof is completed.

\section{Acknowledgements}

The authors would like to express their sincere thanks to the anonymous referees for their time and comments.

\section{Funding}

This work is supported by the National Natural Science Foundation of China under Grant Nos. 11471290, 11871437.

\section{Competing interests}

The authors declare that they have no competing interests.

\section{Authors' contributions}

All authors contributed equally and significantly in writing this paper and typed, read, and approved the final manuscript.

\section{Publisher's Note}

Springer Nature remains neutral with regard to jurisdictional claims in published maps and institutional affiliations.

Received: 10 April 2018 Accepted: 21 January 2019 Published online: 31 January 2019

\section{References}

1. Bates, P., Lu, K., Wang, B.: Attractors of non-autonomous stochastic lattice systems in weighted spaces. Phys. D, Nonlinear Phenom. 289, 32-50 (2014)

2. Shirikyan, A., Zelik, S.: Exponential attractors for random dynamical systems and applications. Stoch. Partial Differ. Equ., Anal. Computat. 1, 241-281 (2013)

3. Zhou, S.: Random exponential attractor for cocycle and application to non-autonomous stochastic lattice systems with multiplicative white noise. J. Differ. Equ. 263, 2247-2279 (2017)

4. Chueshov, I.: Monotone Random Systems Theory and Applications. Springer, New York (2002)

5. Han, X.: Random attractors for second order stochastics lattice dynamical systems with multiplicative noise in weighted spaces. Stoch. Dyn. 12, 1-20 (2012)

6. Han, X.: Asymptotic behaviors for second order stochastic lattice dynamical systems on $\mathbb{Z}^{k}$ in weighted spaces. J. Math. Anal. Appl. 397, 242-254 (2013)

7. Wang, X., Li, S., Xu, D.: Random attractors for second-order stochastic lattice dynamical systems. Nonlinear Anal. 72, 483-494 (2010)

8. Wang, Y., Huang, Z., Alsaadi, F.E.: Existence of random attractors for a class of second-order lattice dynamical systems with Brownian motions. Math. Probl. Eng. 1, 1-13 (2014)

9. Zhou, S., Wei, L.: A random attractor for a stochastic second order lattice system with random coupled coefficients. J. Math. Anal. Appl. 395, 42-55 (2012)

10. Wang, Z., Zhou, S.: Existence and upper semicontinuity of attractors for non-autonomous stochastic lattice FitzHugh-Nagumo systems in weighted spaces. Adv. Differ. Equ. 2016, 310 (2016)

11. Wang, P., Huang, Y., Wang, X.: Random attractors for stochastic discrete complex non-autonomous Ginzburg-Landau equations with multiplicative noise. Adv. Differ. Equ. 2015, 236 (2015)

12. Zhao, X., Li, Y.: Random attractors for the stochastic damped Klein-Gordon-Schrödinger system. Adv. Differ. Equ. $2015,115(2015)$ 
13. Zhou, S., Zhao, M.: Fractal dimension of random attractor for stochastic damped wave equation with multiplicative noise. Discrete Contin. Dyn. Syst. 36, 2887-2914 (2016)

14. Zhou, S., Shi, W.: Attractors and dimension of dissipative lattice systems. J. Differ. Equ. 224, 172-204 (2006)

15. Arnold, L.: Random Dynamical Systems. Springer, Berlin (1998)

16. Fan, X: Attractors for a damped stochastic wave equation of sine-Gordon type with sublinear multiplicative noise. Stoch. Anal. Appl. 24, 767-793 (2006)

Submit your manuscript to a SpringerOpen ${ }^{\circ}$ journal and benefit from:

- Convenient online submission

$\checkmark$ Rigorous peer review

Open access: articles freely available online

- High visibility within the field

- Retaining the copyright to your article

Submit your next manuscript at $\gg$ springeropen.com 